\title{
Algebraic Approach to Entanglement and Entropy
}

\author{
A. P. Balachandran* \\ Institute of Mathematical Sciences, CIT Campus, \\ Taramani, Chennai 600113, India and \\ Physics Department, Syracuse University, Syracuse, NY, 13244-1130, USA \\ T. R. Govindarajan \\ Institute of Mathematical Sciences, CIT Campus, \\ Taramani, Chennai 600113, India and \\ Chennai Mathematical Institute, H1, SIPCOT IT Park, \\ Kelambakkam, Siruseri 603103, India \\ Amilcar R. de Queiro周 \\ Instituto de Fisica, Universidade de Brasilia, \\ Caixa Postal 04455, 70919-970, Brasilia, DF, Braziß \\ A. F. Reyes-Lega \\ Departamento de Física, Universidad de los Andes, \\ A.A. 4976, Bogotá D.C., Colombid
}

(Dated: February 7, 2013) 


\begin{abstract}
We present a general approach to quantum entanglement and entropy that is based on algebras of observables and states thereon. In contrast to more standard treatments, Hilbert space is an emergent concept, appearing as a representation space of the observable algebra, once a state is chosen. In this approach, which is based on the Gel'fand-Naimark-Segal construction, the study of subsystems becomes particularly clear. We explicitly show how the problems associated with partial trace for the study of entanglement of identical particles are readily overcome. In particular, a suitable entanglement measure is proposed, that can be applied to systems of particles obeying Fermi, Bose, para- and even braid group statistics. The generality of the method is also illustrated by the study of time evolution of subsystems emerging from restriction to subalgebras. Also, problems related to anomalies and quantum epistemology are discussed.
\end{abstract}

\footnotetext{
* balachandran38@gmail.com

$\dagger$ trg@imsc.res.in

$\ddagger \overline{\text { amilcarq@unb.br }}$

$\S$ Institute of Mathematical Sciences, CIT Campus, Taramani, Chennai 600113, India

ฯ anreyes@uniandes.edu.co, http://fisicateorica.uniandes.edu.co/anreyes/
} 


\section{INTRODUCTION}

\section{A. On States and Algebras}

In a physical theory, the theoretical description of observations contains two ingredients. The first is the state of the system being observed, it contains the data specifying the system. The second is the specific observable of the system being measured. The output of observations involves a suitable pairing of the state to the observable which yields a number.

In both classical and quantum physics, the state $\omega$ provides a probability distribution and observables form an (associative) algebra.

In classical physics, the state $\omega_{c}$ gives a probability distribution in phase space whereas the algebra $\mathcal{A}_{c}$ of observables consists of real-valued functions on phase space ${ }^{1}$. The product of two functions $\alpha, \beta$ on phase space is point-wise multiplication: for a point $x=(q, p)$ in phase space, $(\alpha \beta)(x)=\alpha(x) \beta(x)$. The algebra $\mathcal{A}_{c}$ is commutative.

The pairing of $\omega_{c}$ to $\alpha$ produces the mean value of $\alpha$, as follows. The mean value of $\alpha$ for the state $\omega_{c}$ is

$$
\begin{aligned}
\omega_{c}(\alpha) & =\int d \mu(x) \rho_{c}(x) \alpha(x) \\
d \mu(p) & =\text { Liouville measure on phase space, }
\end{aligned}
$$

and $\rho_{c}$ is the probability density on phase space associated with $\omega_{c}$.

We note that being a probability density, $\rho_{c}$ is normalised as

$$
\omega_{c}(\mathbb{1}) \equiv \int d \mu(x) \rho_{c}(x)=1
$$

where $\mathbb{1}$ is the constant function with value 1 :

$$
\mathbb{1}(x)=1
$$

In quantum theory we still have a state $\omega$ and an algebra $\mathcal{A}$. The state $\omega$ on an observable $\alpha \in \mathcal{A}$ is generally representable in terms of a density matrix $\rho_{\omega}$ and an operator $\pi_{\omega}(\alpha)$ representing $\alpha$ on a Hilbert space. The mean value of the observable $\alpha$ is then

$$
\omega(\alpha)=\operatorname{Tr}\left(\rho_{\omega} \alpha\right) \equiv \operatorname{Tr}\left(\rho_{\omega} \pi_{\omega}(\alpha)\right)
$$

\footnotetext{
1 More generally one talks of observables as "hermitean" functions on a complexified phase space.
} 
From now on, we represent $\pi_{\omega}(\alpha)$ by $\alpha$ itself, if there is no ambiguity. The state and its density matrix are normalised just as in the classical case:

$$
\omega(\mathbb{1})=\operatorname{Tr} \rho_{\omega}=1 .
$$

The basic mathematical difference between classical and quantum physics lies in this: classical physics is a probability theory on a commutative algebra $\mathcal{A}_{c}$, quantum physics is a probability theory on a non-commutative algebra $\mathcal{A}$.

A state $\omega$ need not be presented using a density matrix to extract numbers from theory. It is enough that $\omega$ is a linear map from $\mathcal{A}$ to $\mathbb{C}$ with the properties

$$
\omega(\mathbb{1})=1, \quad \omega\left(\alpha^{*}\right)=\overline{\omega(\alpha)}, \quad \omega\left(\alpha^{*} \alpha\right) \geq 0 \quad \text { for all } \alpha \text { in } \mathcal{A},
$$

where "*" is a hermitean conjugation ("anti-linear involution") $\mathcal{A}$ should have. A state is best understood in this manner. State vectors and Hilbert spaces play no role at this point. Where, then, do they come from?

\section{B. On the GNS Construction}

State vectors and Hilbert spaces are best thought of as emergent concepts in quantum physics. The primary concepts are states $\omega$ and the algebra $\mathcal{A}$ of observables.

In the 1940's, Gel'fand, Naimark and Segal described the reconstruction of the Hilbert space $\mathcal{H}_{\omega}$ from the data $(\mathcal{A}, \omega)$. The algebra $\mathcal{A}$ acts by a representation $\pi_{\omega}$ on $\mathcal{H}_{\omega}$. This

reconstruction, known as the GNS construction, has played a foundational role in the theory of operator algebras. It has also been an important tool for studies in quantum field theory [1].

We suggest in this paper that the GNS construction is the proper framework for the study of entanglement as well. A brief account of our ideas has appeared before [2].

In section II, we describe the GNS reconstruction of $\left(\mathcal{H}_{\omega}, \pi_{\omega}\right)$ from $(\mathcal{A}, \omega)$. We do not aspire to rigour but to concepts and computations. Whatever we say is correct in finite dimensions. But our presentation omits the fine points of topology for infinite dimensional $\mathcal{A}$.

The GNS construction presents the state $\omega$ as a density matrix $\rho$. We can expand $\rho$ in terms of orthogonal rank 1 density matrices $\rho_{i}$ :

$$
\rho_{i} \rho_{j}=\delta_{i j} \rho_{i}, \quad \operatorname{Tr} \rho_{i}=1,
$$


and accordingly write

$$
\rho=\sum_{i} \lambda_{i} \rho_{i}, \quad \omega=\sum_{i} \lambda_{i} \omega_{i}, \quad \lambda_{i}>0, \quad \sum_{i} \lambda_{i}=1
$$

where $\omega_{i}$ is the state associated with the density matrix $\rho_{i}$. The von Neumann entropy for $\omega$ is then

$$
S(\omega)=-\operatorname{Tr} \rho \log \rho=-\sum_{i} \lambda_{i} \log \lambda_{i} .
$$

We can thus associate an entropy to a pair $(\mathcal{A}, \omega)$ of a state and an algebra of observables. This result is important for us.

\section{What is entanglement}

For a system of non-identical constituents $A_{i}$ with Hilbert spaces $\mathcal{H}_{i}$, 'entanglement' can be understood in terms of 'partial trace' as follows.

It is enough to consider a bipartite system and the Hilbert space

$$
\mathcal{H}=\mathcal{H}_{1} \otimes \mathcal{H}_{2}
$$

Then a density matrix

$$
\rho_{12}=|\psi\rangle\langle\psi|
$$

with a normalised vector state $|\psi\rangle \in \mathcal{H}$ is entangled when the density matrix

$$
\rho_{i}=\operatorname{Tr}_{j} \rho_{12} \quad(j, i=1,2, \quad i \neq j)
$$

obtained by partial tracing has non-zero von Neumann entropy $S\left(\rho_{i}\right)$ :

$$
S\left(\rho_{i}\right)=-\operatorname{Tr} \rho_{i} \log \rho_{i} \neq 0 .
$$

There is no entanglement if $S\left(\rho_{i}\right)=0$.

In section III, we recall that partial tracing has poor physical meaning for systems of identical particles. That is evident from the impressive volume of inconclusive literature on fermions and bosons, and the apparent absence of any treatment of para- and braidstatistics [3-12].

It is thus desirable to replace "partial tracing" by better concepts to study entanglement.

In this section we reformulate the notion of partial tracing in terms of the restriction $\omega_{0}:=\left.\omega\right|_{\mathcal{A}_{0}}$ of a state $\omega$ on an algebra $\mathcal{A}$ to a subalgebra $\mathcal{A}_{0}$. The example we choose is very 
simple: the bipartite system of non-identical particles. This restatement is possible whenever partial tracing can be understood in terms of a restriction. When such interpretation is not possible, partial tracing loses meaning. But the restriction of $\omega$ to subalgebras is always sensible.

The notion of "local operation" (as used in quantum information theory) is implemented here through the choice of an appropriate subalgebra $\mathcal{A}_{0} \subseteq \mathcal{A}$. Importance of focusing on subsystems using subalgebras has also been emphasized in [13-16]. In our approach, the restriction of a state $\omega$ on $\mathcal{A}$ to the subalgebra $\mathcal{A}_{0}$ will give rise to a "reduced", or restricted state $\omega_{0}$, whose entropy compared to that of $\omega$ provides a measure of the entanglement of $\mathcal{A}_{0}$ with $\mathcal{A}$ in the state $\omega$.

For the purposes of this paper, this is what is meant by entanglement.

If $\omega$ is pure, that is, $S(\omega)=0$, the entropy of entanglement is obtained from the expansion

$$
\omega_{0}=\sum_{i} \lambda_{i} \omega_{0, i} \quad \lambda_{i}>0 \quad \sum_{i} \lambda_{i}=1
$$

of $\omega_{0}$ in terms of pure states and using the formula (I.10). There are orderly ways to calculate the expansion (I.9) and that will be taken up in the following sections.

If $\omega$ itself is not pure, we can compare its entropy with that of $\omega_{0}$.

\section{Examples}

We illustrate the GNS approach to entropy by several examples in section IV. The first is simple and involves just the matrix algebra $\mathcal{A}=M_{2}(\mathbb{C})$.

This example is followed up using those from identical fermions and bosons. We clarify what is meant by observing single particle operators of a boson or a fermion in a two-particle system. For this purpose, we need at the simplest level that the single-particle algebra $\mathcal{A}$ is Hopf with a coproduct $\Delta$ compatible with the permutation group which here defines statistic: $2^{2}[17$. This $\Delta$ allows us to identify the correct 'single particle' subalgebra $\Delta(\mathcal{A})$ of the full two-particle algebra and calculate $\left.\omega\right|_{\Delta(\mathcal{A})}$ and its entropy for any two-body state $\omega$. It does not agree with what one finds from partial tracing.

We can also illustrate all this for para-bosons and para-fermions. For these cases, $\Delta$ is not changed. So we instead consider the braid group $B_{N}$ to be the $N$-particle statistics group.

\footnotetext{
${ }^{2}$ It is perhaps enough if $\mathcal{A}$ is quasi-Hopf or just a coalgebra.
} 
In that case $\Delta$ is changed to $\Delta_{b}$. We calculate the restriction of a chosen three-particle state to single-particle observables, obtained from $\Delta_{b}$ in a systematic manner and calculate its entropy. It illustrates the effectiveness of the GNS approach when combined with concepts from Hopf algebras, in quantum information theory.

If the subalgebra $\mathcal{A}_{0}$ comes from a $k$-particle subsystem in an $N$-particle system, it is necessary that $\mathcal{A}_{0}$ is at least a coalgebra with a coproduct $\Delta$ to extend its meaning beyond $N$-particles. This is a new point of our work.

Second quantization also requires the notion of coproduct [17].

\section{E. Time evolution}

In the GNS-approach, given the time evolution $\omega(t)$ of a state $\omega \equiv \omega(0)$ on $\mathcal{A}$, it induces a time evolution on the restriction $\omega_{0}=\left.\omega\right|_{\mathcal{A}_{0}}$ of $\omega$ to $\mathcal{A}_{0}$. It is

$$
\omega_{0}(t)=\left.\omega(t)\right|_{\mathcal{A}_{0}}
$$

If $\omega(0)$ is represented by a density matrix $\rho(0)$ with unitary time evolution according to

$$
\begin{aligned}
\rho(t) & =U(t)^{-1} \rho(0) U(t), \\
U(t) & =e^{-i t H}, \quad H=\text { Hamiltonian, }
\end{aligned}
$$

there is no assurance that $\rho_{0}(t)=\left.\rho(t)\right|_{\mathcal{A}_{0}}$ also undergoes a unitary evolution.

The generic situation will rather be that of an evolution by positive maps. This follows from Stinespring's theorem [18].

We illustrate the evolution $\rho_{0}(t) \rightarrow \rho_{0}(t+\tau)$ by completely positive maps using our algebraic approach, in section V. Here we only remark that this type of evolution arises very naturally from the restriction to a subalgebra. This is the basic mechanism behind quantum decoherence.

\section{F. Anomalies}

It has been suggested [19, 20] that unwanted anomalies, such as the $T$-violation associated with the QCD $\theta$-angle, can be eliminated by using mixed states. The appearance of such mixed states for restoring anomalies can also be explained in terms of restrictions of states 
to subalgebras, as we show in section VI. This argument provides further strong evidence for the power of the proposition for the use of mixed states in that context.

The GNS theory is thus useful in many different physical contexts.

\section{G. On state restrictions and quantum epistemology}

We suggest in section VII of this paper that the restriction $\omega_{0}=\left.\omega\right|_{\mathcal{A}_{0}}$ of a state $\omega$ on $\mathcal{A}$ can also be understood in terms of "collapse of wave packets" and an interpretative superstructure of quantum theory. Thus suppose that we have an algebra $\mathcal{A}$ of observables acting on a Hilbert space $\mathcal{H}$ and we observe a projector $p \in \mathcal{A}$. Let $\mathcal{A}_{0} \subset \mathcal{A}$ be the maximal subalgebra which commutes with $p$ (and hence includes $p$ ). Then we can interpret $\omega_{0}$ as the restriction of $\omega$ to $\mathcal{A}_{0}$. We explain how this is so in that section.

\section{THE GNS CONSTRUCTION}

For completeness, we begin with a few definitions. They are all effortlessly fulfilled in all our examples where we consider only finite-dimensional matrix algebras.

A $*$-algebra $\mathcal{A}$ is an associative algebra over $\mathbb{C}$ with an anti-linear involution ("hermitean conjugation"):

$$
\begin{aligned}
*: \mathcal{A} & \rightarrow \mathcal{A} \\
*^{2} & =\mathrm{id} .
\end{aligned}
$$

A $C^{*}$-norm $\|\cdot\|$ on such an algebra $\mathcal{A}$ is a norm fulfilling the property

$$
\left\|\alpha^{*} \alpha\right\|=\|\alpha\|^{2}, \quad \forall \alpha \in \mathcal{A} .
$$

If it exists, it is unique.

Algebras $M_{d}(\mathbb{C})$ of $d \times d$ matrices can all be regarded as $C^{*}$-algebras, with the norm fixed by

$$
\|\alpha\|^{2}=\text { largest eigenvalue of } \alpha^{*} \alpha .
$$

The algebra $\mathcal{A}$ of observables in quantum theory is a $C^{*}$-algebra. Indeed, the algebra of all bounded operators $\mathcal{B}(\mathcal{H})$ on a separable Hilbert space admits a norm to turn it into a $C^{*}$-algebra. 
We will assume that $\mathcal{A}$ is unital with unity $\mathbb{1}_{\mathcal{A}}$. That is needed to discuss completeness relations, for example.

The data we are given is thus $(\mathcal{A}, \omega)$. We can construct $\left(\mathcal{H}_{\omega}, \pi_{\omega}(\mathcal{A})\right)$ from this data as follows.

For each element $\alpha \in \mathcal{A}$, we associate a vector $|\alpha\rangle$ in a complex vector space $\hat{\mathcal{A}}$ with the property

$$
\begin{gathered}
|\lambda \alpha+\mu \beta\rangle=\lambda|\alpha\rangle+\mu|\beta\rangle, \\
\lambda, \mu \in \mathbb{C} ; \alpha, \beta \in \mathcal{A} .
\end{gathered}
$$

Next, an inner product is introduced in $\hat{\mathcal{A}}$ using $\omega$ :

$$
\langle\beta \mid \alpha\rangle=\omega\left(\beta^{*} \alpha\right)
$$

It fulfills

$$
\left\langle\mathbb{1}_{\mathcal{A}} \mid \alpha\right\rangle=\omega(\alpha)=\overline{\left\langle\alpha \mid \mathbb{1}_{\mathcal{A}}\right\rangle}=\overline{\omega\left(\alpha^{*}\right)}
$$

and Schwarz inequality as well in view of $(\mathrm{I} .7)\left(\omega\left(\alpha^{*} \alpha\right) \geq 0\right)$ :

$$
|\langle\beta \mid \alpha\rangle|^{2} \leq\langle\beta \mid \beta\rangle\langle\alpha \mid \alpha\rangle
$$

But it may not be a scalar product from which we can build a Hilbert space, as there may be $0 \neq \alpha \in \mathcal{A}$ giving vectors $|\alpha\rangle$ of zero norm:

$$
\langle\alpha \mid \alpha\rangle=0
$$

Let $\mathcal{N}_{\omega}$ denote the subspace of $\mathcal{A}$ whose image $\hat{\mathcal{N}}_{\omega} \subset \hat{\mathcal{A}}$ are vectors of zero norm:

$$
\mathcal{N}_{\omega}=\{\alpha \in \mathcal{A} \mid\langle\alpha \mid \alpha\rangle=0\}
$$

Observe that, from Schwarz inequality,

$$
\langle a \mid \alpha\rangle=0, \forall a \in \mathcal{A}, \alpha \in \mathcal{N}_{\omega}
$$

Hence, $\mathcal{N}_{\omega}$ is a left-ideal. That is,

$$
a \mathcal{N}_{\omega} \subseteq \mathcal{N}_{\omega} \quad \forall a \in \mathcal{A}
$$

This follows from (II.10): if $a \in \mathcal{A}$ and $\alpha \in \mathcal{N}_{\omega},\langle a \alpha \mid a \alpha\rangle=\left\langle a^{*} a \alpha \mid \alpha\right\rangle=0$ by (II.10). The subspace $\mathcal{N}_{\omega}$ is called the Gel'fand ideal. 
We next consider the vector space

$$
\hat{\mathcal{A}} / \hat{\mathcal{N}}_{\omega}=\left\{|[a]\rangle:=\left|a+\mathcal{N}_{\omega}\right\rangle, \quad a \in \mathcal{A}\right\}
$$

The label $[a]$ of a vector denotes an equivalence class $a+\mathcal{N}_{\omega}$, a set, in $\mathcal{A}$.

Now,

a) $\hat{\mathcal{A}} / \hat{\mathcal{N}}_{\omega}$ has a well-defined scalar product $\langle\cdot \mid \cdot\rangle$ given by

$$
\langle[a] \mid[b]\rangle=\omega\left(a^{*} b\right) .
$$

In particular the vector $\left|\mathcal{N}_{\omega}\right\rangle$, playing the role of zero, is the only vector that has zero norm. Note in this connection that since $\omega\left(a^{*}(b+\alpha)\right)=\omega\left(a^{*} b\right)$ for all $\alpha \in \mathcal{N}_{\omega}$, the right hand side of (II.13) does not depend on the choices of representatives $a, b$ from $[a]$ and $[b]$.

We denote the Hilbert space obtained by the closure of $\hat{\mathcal{A}} / \hat{\mathcal{N}}_{\omega}$ under II.13) as $\mathcal{H}_{\omega}$.

b) Because of (II.11), $\mathcal{H}_{\omega}$ carries a representation $\pi_{\omega}$ of $\mathcal{A}$ :

$$
\pi_{\omega}(a)|[b]\rangle:=|[a b]\rangle
$$

We have now obtained $\left(\mathcal{H}_{\omega}, \pi_{\omega}(\mathcal{A})\right)$ from $(\mathcal{A}, \omega)$ thereby completing the GNS construction.

\section{A. Properties of the GNS Representation}

Consider the vector $\left|\left[\mathbb{1}_{\mathcal{A}}\right]\right\rangle$. Then, if $\pi_{\omega}(\mathcal{A})$ denotes the set $\left\{\pi_{\omega}(a): \forall a \in \mathcal{A}\right\}$,

$$
\pi_{\omega}(\mathcal{A})\left|\left[\mathbb{1}_{\mathcal{A}}\right]\right\rangle=\{|[a]\rangle \mid a \in \mathcal{A}\}
$$

so that from $\left|\left[\mathbb{1}_{\mathcal{A}}\right]\right\rangle$ we can generate all vectors of $\mathcal{H}_{\omega}$ by acting with $\pi_{\omega}(\mathcal{A})$ and closure. Such a vector $\left|\left[\mathbb{1}_{\mathcal{A}}\right]\right\rangle$ is said to be cyclic. The representation $\pi_{\omega}$ is a cyclic representation.

The state $\omega$ can now be represented as a density matrix $\rho_{\omega}$,

$$
\rho_{\omega}=\left|\left[\mathbb{1}_{\mathcal{A}}\right]\right\rangle\left\langle\left[\mathbb{1}_{\mathcal{A}}\right]\right|
$$

That is because

$$
\begin{aligned}
\omega\left(a^{*} b\right) & =\langle[a] \mid[b]\rangle=\left\langle\left[\mathbb{1}_{\mathcal{A}}\right]\left|\pi_{\omega}\left(a^{*}\right) \pi_{\omega}(b)\right|\left[\mathbb{1}_{\mathcal{A}}\right]\right\rangle \\
& =\operatorname{Tr}\left(\rho_{\omega} \pi_{\omega}\left(a^{*}\right) \pi_{\omega}(b)\right) .
\end{aligned}
$$




\section{B. On irreducibility and entropy}

The representation $\pi_{\omega}$ may not be irreducible. In finite dimensions at least, which are our concern here, it can be reduced to a direct sum of irreducible representations (IRR's) $\pi_{\omega}^{(\alpha)}$ :

$$
\pi_{\omega}=\oplus_{\alpha} \pi_{\omega}^{(\alpha)} .
$$

That is because $\mathcal{A}$ is a $*$-algebra. The proof is similar to the one for finite groups $G$, their group algebras also being $*$-algebras.

The proof of II.18 goes as follows. If $\mathcal{H}_{\omega}^{(1)} \subset \mathcal{H}_{\omega}$ is a non-trivial invariant subspace under $\pi_{\omega}(\mathcal{A})$, then so is its orthogonal complement $\mathcal{H}_{\omega}^{(1) \perp}$. For if

$$
|[\alpha]\rangle \in \mathcal{H}_{\omega}^{(1)}, \quad|[\beta]\rangle \in \mathcal{H}_{\omega}^{(1) \perp},
$$

so that $\langle[\alpha] \mid[\beta]\rangle=0$, then

$$
\langle[\alpha] \mid[a \beta]\rangle=0, \quad \forall a \in \mathcal{A},
$$

for the left-hand side is $\left\langle\left[a^{*} \alpha\right] \mid[\beta]\right\rangle$. But $a^{*} \in \mathcal{A}(\mathcal{A}$ being $*)$ and hence $\left|\left[a^{*} \alpha\right]\right\rangle \in \mathcal{H}_{\omega}^{(1)}$. The statement follows.

Now, $\mathcal{H}_{\omega}^{(1)}$ (and similarly $\mathcal{H}_{\omega}^{(1) \perp}$ ) is either irreducible or has a non-trivial invariant subspace. If the latter is the case, we repeat the process ending up with

$$
\mathcal{H}_{\omega}=\oplus_{\alpha} \mathcal{H}_{\omega}^{(\alpha)},
$$

where the sum is the orthogonal direct sum and $\mathcal{H}_{\omega}^{(\alpha)}$ carries $\pi_{\omega}^{(\alpha)}(\mathcal{A})$.

We now show how to correspondingly decompose $\rho_{\omega}$ into a convex sum of orthogonal rank 1 density matrices:

$$
\begin{aligned}
\rho_{\omega} & =\sum_{\alpha} \lambda_{\alpha} \rho_{\omega}^{(\alpha)}, \quad \lambda_{\alpha}>0, \quad \sum_{\alpha} \lambda_{\alpha}=1, \\
\rho_{\omega}^{(\alpha)} \rho_{\omega}^{(\beta)} & =\delta_{\alpha \beta} \rho_{\omega}^{(\alpha)} .
\end{aligned}
$$

For this purpose, we write

$$
\left|\left[\mathbb{1}_{\mathcal{A}}\right]\right\rangle=\sum_{\alpha}\left|\left[\mathbb{1}_{\mathcal{A}}^{(\alpha)}\right]\right\rangle, \quad\left|\left[\mathbb{1}_{\mathcal{A}}^{(\alpha)}\right]\right\rangle \in \mathcal{H}_{\omega}^{(\alpha)},
$$

that is, we decompose the left hand side into its components in $\mathcal{H}_{\omega}^{(\alpha)}$. Now, since for $\alpha \neq \beta$

$$
\left\langle\left[\mathbb{1}_{\mathcal{A}}^{(\alpha)}\right]\left|\pi_{\omega}(a)\right|\left[\mathbb{1}_{\mathcal{A}}^{(\beta)}\right]\right\rangle=0,
$$


the density matrix $\rho_{\omega}$ can be rewritten as

$$
\rho_{\omega}=\sum_{\alpha}\left|\left[\mathbb{1}_{\mathcal{A}}^{(\alpha)}\right]\right\rangle\left\langle\left[\mathbb{1}_{\mathcal{A}}^{(\alpha)}\right]\right|
$$

If we set

$$
\lambda_{\alpha}=\left\langle\left[\mathbb{1}_{\mathcal{A}}^{(\alpha)}\right] \mid\left[\mathbb{1}_{\mathcal{A}}^{(\alpha)}\right]\right\rangle
$$

we can rewrite $\rho_{\omega}$ in terms of normalised vectors, as follows. We define

$$
\left|\chi^{(\alpha)}\right\rangle=\frac{1}{\sqrt{\lambda_{\alpha}}}\left|\left[\mathbb{1}_{\mathcal{A}}^{(\alpha)}\right]\right\rangle
$$

Then,

$$
\left\langle\chi^{(\alpha)} \mid \chi^{(\beta)}\right\rangle=\delta_{\alpha \beta}
$$

With

$$
\rho_{\omega}^{(\alpha)}:=\left|\chi^{(\alpha)}\right\rangle\left\langle\chi^{(\alpha)}\right|
$$

we then obtain the decomposition of $\rho_{\omega}$ in terms of pure states as

$$
\rho_{\omega}=\sum_{\alpha} \lambda_{\alpha} \rho_{\omega}^{(\alpha)}
$$

where

$$
\lambda_{\alpha}>0, \quad \sum_{\alpha} \lambda_{\alpha}=1, \quad \rho_{\omega}^{(\alpha)} \rho_{\omega}^{(\beta)}=\delta_{\alpha \beta} \rho_{\omega}^{(\alpha)} .
$$

The von Neumann entropy of $\rho_{\omega}$ is

$$
S\left(\rho_{\omega}\right)=-\operatorname{Tr} \rho_{\omega} \log \rho_{\omega}=-\sum_{\alpha} \lambda_{\alpha} \log \lambda_{\alpha}
$$

Corresponding to II.30, we have the decomposition of $\omega$ into extremal or pure states $\omega^{(\alpha)}$ (Recall that $\omega(\cdot)=\operatorname{Tr} \rho \cdot)$ :

$$
\omega=\sum_{\alpha} \lambda_{\alpha} \omega^{(\alpha)}
$$

It has entropy

$$
S(\omega)=-\sum_{\alpha} \lambda_{\alpha} \log \lambda_{\alpha}
$$

There are important issues related to the uniqueness of the decomposition (II.30) and hence of the entropy of $\omega$ as observed by R. Sorkin [21] [cf. eq. (IV.27)]. For a detailed discussion of these issues we refer to [22]. 


\section{ON ENTANGLEMENT AND SUBALGEBRAS}

For a bipartite system of non-identical particles $A$ and $B$ with Hilbert spaces $\mathcal{H}_{A}$ and $\mathcal{H}_{B}$, a vector state $|\psi\rangle \in \mathcal{H}=\mathcal{H}_{A} \otimes \mathcal{H}_{B}$, which in general is of the form

$$
|\psi\rangle=\sum_{i, j} C_{i j}\left|\chi_{A, i}\right\rangle \otimes\left|\eta_{B, j}\right\rangle
$$

is said to be entangled if it cannot be reduced to the form

$$
|\psi\rangle=\left|\chi_{A}^{\prime}\right\rangle \otimes\left|\eta_{B}^{\prime}\right\rangle
$$

by a change of basis.

A measure of entanglement is the von Neumann entropy of the reduced density matrix

$$
\rho_{A}=\operatorname{Tr}_{\mathcal{H}_{B}}|\psi\rangle\langle\psi|
$$

We assume that $\langle\psi \mid \psi\rangle=1$. The vector $|\psi\rangle$ is entangled if and only if

$$
S\left(\rho_{A}\right)=-\rho_{A} \log \rho_{A} \neq 0 .
$$

The physical meaning of the partial trace and the reduced density matrix are as follows. Suppose that we observe only the subalgebra

$$
\mathcal{A}_{0}=\left\{\alpha_{0} \in \mathcal{A} \mid \alpha_{0}=K_{A} \otimes \mathbb{1}_{B}\right\}
$$

where $K_{A}$ is an observable acting on $\mathcal{H}_{\mathcal{A}}$ and $\mathbb{1}_{B}$ is the identity operator on $\mathcal{H}_{B}$. The algebra $\mathcal{A}$ of all observables on $\mathcal{H}=\mathcal{H}_{A} \otimes \mathcal{H}_{B}$ contains $\mathcal{A}_{0}$ as a subalgebra:

$$
\mathcal{A}_{0} \subset \mathcal{A}
$$

Now, for restricted observations of just $\mathcal{A}_{0}$,

$$
\operatorname{Tr}_{\mathcal{H}}\left(\rho \alpha_{0}\right)=\operatorname{Tr}_{\mathcal{H}_{A}}\left(\rho_{A} K_{A}\right), \quad \alpha_{0}=K_{A} \otimes \mathbb{1}_{B} \in \mathcal{A}_{0}
$$

Thus, $\rho_{A}$ is said to be the restriction of $\rho$ to $\mathcal{A}_{0}$.

Let $\omega$ be the state for the density matrix $\rho=|\psi\rangle\langle\psi|$ :

$$
\omega(\alpha)=\operatorname{Tr}(\rho \alpha), \quad \alpha \in \mathcal{A} .
$$


In the same way, let $\omega_{A}$ be the state for the density matrix $\rho_{A}$ :

$$
\omega_{A}\left(\alpha_{0}\right)=\operatorname{Tr}_{\mathcal{H}_{A}}\left(\rho_{A} K_{A}\right), \quad \alpha_{0}=K_{A} \otimes \mathbb{1}_{B} \in \mathcal{A}_{0}
$$

Then $\omega_{A}$ is said to be the restriction of the state $\omega$ on $\mathcal{A}$ to $\mathcal{A}_{0}$ :

$$
\omega_{A}=\left.\omega\right|_{\mathcal{A}_{0}} .
$$

Thus partial trace in this case maps a density matrix $\rho$ and a state $\omega$ on $\mathcal{A}$ to their restrictions $\rho_{A}, \omega_{A}$ on $\mathcal{A}_{0}$.

But there are many cases where partial trace cannot be interpreted this way and in fact has no physical meaning. A well-discussed example is that of identical fermions [5, 8, 10, 11]. Denoting antisymmetrisation by $\wedge$,

$$
|\psi\rangle \wedge|\chi\rangle=\frac{1}{\sqrt{2}}(|\psi\rangle \otimes|\chi\rangle-|\chi\rangle \otimes|\psi\rangle),
$$

a generic $N$-particle vector of identical fermions is a linear combination of vectors of the form

$$
|\psi\rangle=\left|\psi_{1}\right\rangle \wedge\left|\psi_{2}\right\rangle \wedge \ldots \wedge\left|\psi_{N}\right\rangle .
$$

It lives in the $N$-fold antisymmetric product $\mathcal{H}$ of the one-particle Hilbert space $\mathcal{H}^{(1)}$ :

$$
\mathcal{H}=\bigwedge^{N} \mathcal{H}^{(1)}, \quad|\psi\rangle \in \mathcal{H} .
$$

The algebra $\mathcal{A}$ of observables must necessarily leave $\mathcal{H}$ invariant. That means that observables must be permutation invariant. An operator such as $K_{1} \otimes \mathbb{1} \otimes \cdots \otimes \mathbb{1}$ (1 is the identity on $\mathcal{H}^{(1)}$ and $K_{1} \neq \mathbb{1}$ ) is not permutation invariant and, therefore, is not an observable. Hence now partial traces do not correspond to restrictions to subalgebras of observables on $\mathcal{H}$.

So, in a generic problem, partial trace is not a meaningful operation.

But the restriction of a state $\omega$ on $\mathcal{A}$ to a subalgebra $\mathcal{A}_{0}$ is always sensible. What we need is a criterion to select $\mathcal{A}_{0}$ appropriately for a physical question.

For example, the algebra $\mathcal{A}_{0}$ appropriate for single particle observables is generated by

$$
K \otimes \mathbb{1} \otimes \cdots \otimes \mathbb{1}+\mathbb{1} \otimes K \otimes \cdots \otimes \mathbb{1}+\cdots+\mathbb{1} \otimes \cdots \otimes \mathbb{1} \otimes K
$$

where $K$ is an observable on $\mathcal{H}^{(1)}$. 
Choices such as (III.14) are dictated by a coproduct on the single-particle algebra. We will return to this point later.

For such reasons, as declared in the Introduction, entanglement is a property characterising a triple $\left(\mathcal{A}, \mathcal{A}_{0}, \omega\right)$. We avoid the use of partial trace.

\section{EXAMPLES}

\section{A. The Algebra $M_{2}(\mathbb{C})$}

The choice $\mathcal{A}=M_{2}(\mathbb{C})$ of $2 \times 2$ complex matrices is a simple non-trivial example to illustrate the GNS construction. It is discussed already in appendix 3 of Landi [23]. We will recall this example here and, in addition, use it to illustrate the entropy calculation.

The algebra $\mathcal{A}$ acts on $\mathbb{C}^{2}$. Let

$$
\left\{|i\rangle: i=1,2,\langle i \mid j\rangle=\delta_{i j}\right\}
$$

be an orthonormal basis of $\mathbb{C}^{2}$. Then the matrix units

$$
e_{i j}=|i\rangle\langle j|
$$

span $M_{2}(\mathbb{C})$. Note that

$$
e_{i j} e_{k l}=\delta_{j k} e_{i l}
$$

An element $\alpha$ of $\mathcal{A}$ can be expanded in this basis as

$$
\alpha=\sum_{i, j} \alpha_{i j} e_{i j}
$$

Following [2, for the state $\omega$ we choose

$$
\omega(\alpha)=\lambda \alpha_{11}+(1-\lambda) \alpha_{22}, \quad 0 \leq \lambda \leq 1
$$

The null space $\mathcal{N}_{\omega}$ is determined by the condition

$$
\omega\left(\alpha^{*} \alpha\right)=0
$$

For our choice (IV.5) for $\omega$ we obtain, making use of (IV.3),

$$
\omega\left(\alpha^{*} \alpha\right)=\lambda\left(\left|\alpha_{11}\right|^{2}+\left|\alpha_{21}\right|^{2}\right)+(1-\lambda)\left(\left|\alpha_{12}\right|^{2}+\left|\alpha_{22}\right|^{2}\right) .
$$


The solution depends on $\lambda$. We consider 3 cases.

Case 1: $\lambda=0$.

In this case, $\alpha \in \mathcal{N}_{\omega}$ if $\alpha_{12}=\alpha_{22}=0$. So

$$
\mathcal{N}_{\omega}=\left\{\left(\begin{array}{ll}
\alpha_{11} & 0 \\
\alpha_{21} & 0
\end{array}\right): \alpha_{11}, \alpha_{21} \in \mathbb{C}\right\} \cong \mathbb{C}^{2} .
$$

Since $\hat{\mathcal{A}} \cong \mathbb{C}^{4}$, we obtain

$$
\mathcal{H}_{\omega}=\hat{\mathcal{A}} / \hat{\mathcal{N}}_{\omega} \cong \mathbb{C}^{2}
$$

with basis

$$
\left\{\left|\left[e_{k 2}\right]\right\rangle\right\}_{k=1,2}
$$

The representation $\pi_{\omega}$ of $\mathcal{A}$ on $\mathcal{H}_{\omega}$ is given by

$$
\pi_{\omega}\left(e_{i j}\right)\left|\left[e_{k 2}\right]\right\rangle=\delta_{j k}\left|\left[e_{i 2}\right]\right\rangle .
$$

It is isomorphic to the defining representation on $\mathbb{C}^{2}$. Therefore it is irreducible. So we conclude that $\rho_{\omega}$ is a rank 1 projector and has vanishing entropy:

$$
S\left(\rho_{\omega}\right)=0
$$

Case 2: $\lambda=1$.

This is similar to the case $\lambda=0$. The null space $\hat{\mathcal{N}}_{\omega} \cong \mathbb{C}^{2}$ is spanned by

$$
\left|e_{12}\right\rangle,\left|e_{22}\right\rangle \text {. }
$$

and $\mathcal{H}_{\omega}=\hat{\mathcal{A}} / \hat{\mathcal{N}}_{\omega}$ has basis

$$
\left\{\left|\left[e_{k 1}\right]\right\rangle\right\}_{k=1,2}
$$

The representation $\pi_{\omega}$ is irreducible, isomorphic to the $\lambda=0$ representation and carries zero entropy.

Case 3: $0<\lambda<1$.

There are no non-zero null vectors in this case:

$$
\mathcal{N}_{\omega_{\lambda}}=\{0\} .
$$

Hence

$$
\mathcal{H}_{\omega}=\hat{\mathcal{A}} / \hat{\mathcal{N}}_{\omega} \cong \mathbb{C}^{4}
$$


It has basis

$$
\left\{\left|\left[e_{i j}\right]\right\rangle\right\}_{i, j=1,2}
$$

The representation $\pi_{\omega}$ is given by

$$
\pi_{\omega}\left(e_{i j}\right)\left|\left[e_{k l}\right]\right\rangle=\left|\left[e_{i j} e_{k l}\right]\right\rangle=\delta_{j k}\left|\left[e_{i l}\right]\right\rangle
$$

This representation is reducible into two two-dimensional irreducible ones: $\mathcal{H}_{\omega}=\mathbb{C}^{2} \oplus \mathbb{C}^{2}$. The first $\mathbb{C}^{2}$ has basis

$$
\left\{e_{a 1}\right\}_{a=1,2}
$$

and the second

$$
\left\{e_{a 2}\right\}_{a=1,2}
$$

We must next express $\left|\left[\mathbb{1}_{\mathcal{A}}\right]\right\rangle$ in terms of its components in these subspaces. That is easy:

$$
\left|\left[\mathbb{1}_{\mathcal{A}}\right]\right\rangle=\left|\left[e_{11}\right]\right\rangle+\left|\left[e_{22}\right]\right\rangle
$$

It follows that $\omega_{\lambda}$ is not pure and can be expressed in terms of the following density matrix:

$$
\rho_{\omega_{\lambda}}=\left|\left[e_{11}\right]\right\rangle\left\langle\left[e_{11}\right]|+|\left[e_{22}\right]\right\rangle\left\langle\left[e_{22}\right]\right| .
$$

Since

$$
\begin{gathered}
\left\langle\left[e_{11}\right] \mid\left[e_{11}\right]\right\rangle=\omega\left(e_{11}^{*} e_{11}\right)=\omega\left(e_{11}\right)=\lambda \\
\left\langle\left[e_{22}\right] \mid\left[e_{22}\right]\right\rangle=\omega\left(e_{22}^{*} e_{22}\right)=\omega\left(e_{22}\right)=1-\lambda, \\
\rho_{\omega_{\lambda}}=\lambda \rho_{11}+(1-\lambda) \rho_{22},
\end{gathered}
$$

where $\rho_{11}$ and $\rho_{22}$ are the rank 1 density matrices

$$
\begin{aligned}
& \rho_{11}=\frac{1}{\lambda}\left|\left[e_{11}\right]\right\rangle\left\langle\left[e_{11}\right]\right|, \\
& \rho_{22}=\frac{1}{1-\lambda}\left|\left[e_{22}\right]\right\rangle\left\langle\left[e_{22}\right]\right| .
\end{aligned}
$$

We can read off the entropy of $\rho_{\omega_{\lambda}}$ to be

$$
S\left(\rho_{\omega_{\lambda}}\right)=-\lambda \log \lambda-(1-\lambda) \log (1-\lambda)
$$

Remark:

This example shows that the irreducible representations of dimension 2 occur with multiplicity 2. This is a general feature: for any representation $\pi$ of a $C^{*}$-algebra $\mathcal{A}$ with a 
cyclic and separating vector ${ }^{3}[1$, an irreducible representation of dimension $d$ occurs with multiplicity $d$. This is as in regular representations of compact groups and follows from Tomita-Takesaki theory [1]

Rafael Sorkin has pointed out to us [21] that if $d>1$, the splitting of $\mathcal{H}_{\omega}$ into irreducible subspaces such as $\mathcal{H}=\mathbb{C}^{2} \oplus \mathbb{C}^{2}$ before $(\mathrm{IV} .19)$ is not unique. For example, we could have chosen another pair of $\mathbb{C}^{2}$ 's with basis

$$
\begin{aligned}
& \sum_{\alpha} \xi_{\alpha}\left|\left[e_{a \alpha}\right]\right\rangle, \quad \sum_{\alpha} \eta_{\alpha}\left|\left[e_{a \alpha}\right]\right\rangle, \\
& \xi^{\dagger} \cdot \xi, \quad \eta^{\dagger} \cdot \eta \neq 0, \quad \xi^{\dagger} \cdot \eta=0
\end{aligned}
$$

and recalculated $\rho_{\omega_{\lambda}}$ and its entropy. They depend on $\xi, \eta$. This feature is generic for $d>1$. The entropy (IV.26) is the least one. Further discussion of such issues will be given elsewhere $[22]$.

\section{B. $\quad$ A $\mathbb{C}^{2} \otimes \mathbb{C}^{2}$ example}

The matrices

$$
\sigma_{\mu}: \quad \sigma_{0}=\mathbb{1}_{2}, \quad \sigma_{i}=\text { Pauli matrices, }
$$

form a basis for $M_{2}(\mathbb{C})$. So the algebra $\mathcal{A}=M_{4}(\mathbb{C})$ on $\mathbb{C}^{2} \otimes \mathbb{C}^{2}$ is generated by

$$
\sigma_{\mu} \otimes \mathbb{1}_{2}, \quad \mathbb{1}_{2} \otimes \sigma_{\mu}, \quad \mu=0, \ldots, 3
$$

For the state $\omega \equiv \omega_{\theta}$, let us choose :

$$
\rho_{\omega_{\theta}}=\left|\psi_{\theta}\right\rangle\left\langle\psi_{\theta}|, \quad| \psi_{\theta}\right\rangle=\cos \theta|+-\rangle-\sin \theta|-+\rangle
$$

Here

$$
|+-\rangle=|+\rangle \otimes|-\rangle, \quad \text { etc. }
$$

with

$$
|+\rangle=\left(\begin{array}{l}
1 \\
0
\end{array}\right), \quad|-\rangle=\left(\begin{array}{l}
0 \\
1
\end{array}\right)
$$

\footnotetext{
${ }^{3}$ A vector state $|\Omega\rangle \in \mathcal{H}$ is cyclic when $\pi(\mathcal{A})|\Omega\rangle$ is dense in $\mathcal{H}$. A state $|\Omega\rangle \in \mathcal{H}$ is separating when the map $\mathcal{A} \rightarrow \pi(\mathcal{A})|\Omega\rangle$ is injective.
} 
Let us now choose, for the subalgebra $\mathcal{A}_{0}$, the subalgebra generated by the ("local") operators

$$
\left\{\sigma_{\mu} \otimes \mathbb{1}_{2}\right\}_{\mu=0, \ldots, 3} .
$$

In this context, entanglement can be understood in terms of correlations between measurements performed by two observers $A$ and $B$ having access to observables corresponding only to $\mathcal{A}_{0}$ (in the case of, say, $A$ ) and observables corresponding only to the commutant of $\mathcal{A}_{0}$ (in the case of $B$ ).

For $A$, the state $\omega_{\theta}$ becomes the restriction of $\omega_{\theta}$ to $\mathcal{A}_{0}$ :

$$
\omega_{\theta, 0}=\left.\omega_{\theta}\right|_{\mathcal{A}_{0}} .
$$

Notice that, in this case, the result of the restriction coincides with the one obtained by partial trace. In fact, since every element of $\mathcal{A}_{0}$ is of the form $\tilde{\alpha}=\alpha \otimes \mathbb{1}_{2}$ (for some $\alpha \in M_{2}(\mathbb{C})$ ), we obtain:

$$
\begin{aligned}
\omega_{\theta, 0}(\tilde{\alpha}) & =\left\langle\psi_{\theta}\left|\left(\alpha \otimes \mathbb{1}_{2}\right)\right| \psi_{\theta}\right\rangle \\
& =\cos ^{2} \theta\langle+|\alpha|+\rangle+\sin ^{2} \theta\langle-|\alpha|-\rangle \\
& =\cos ^{2} \theta \alpha_{11}+\sin ^{2} \theta \alpha_{22}
\end{aligned}
$$

Taking into account the fact that $\mathcal{A}_{0} \cong M_{2}(\mathbb{C})$, and comparing equations (IV.34) and (IV.5), we see that the entropy obtained from the GNS construction is given by (IV.26) upon replacing $\lambda$ by $\cos ^{2} \theta$ :

$$
S(\theta)=-\cos ^{2} \theta \log \cos ^{2} \theta-\sin ^{2} \theta \log \sin ^{2} \theta .
$$

Clearly, $S(\theta)$ corresponds to the entanglement of the vector state $\left|\psi_{\theta}\right\rangle$. In particular, for $\theta=\pi / 4$ the state is the (maximally entangled) Bell vector state

$$
\left|\psi_{\theta=\frac{\pi}{4}}\right\rangle=\frac{1}{\sqrt{2}}(|+-\rangle-|-+\rangle),
$$

for which (IV.35) reduces to $\log 2$.

Now, in order to illustrate how to deal with cases where $\mathcal{A}_{0}$ does not act on just one factor of a bipartite system, we consider the Bell state (IV.36) together with different choices for $\mathcal{A}_{0}$. We focus on choices for which partial trace has no meaning. For instance, we now consider the following three choices: 
- $\mathcal{A}_{ \pm}$, generated by $\left\{\sigma_{\mu} \otimes\left(\frac{1 \pm \sigma_{3}}{2}\right)\right\}_{\mu}$.

- $\mathcal{A}_{+} \oplus \mathcal{A}_{-}$.

(Note that $\mathcal{A}_{+} \mathcal{A}_{-}=\{0\}$ ).

Case 1: $\mathcal{A}_{0}=\mathcal{A}_{+}$.

The null space $\widehat{\mathcal{N}}_{\omega}^{+} \subset \hat{\mathcal{A}}_{+}$is determined by the equation

$$
\rho_{\omega}\left(\alpha^{*} \alpha\right)=\left\langle\psi_{\theta=\frac{\pi}{4}}\left|\alpha^{*} \alpha\right| \psi_{\theta=\frac{\pi}{4}}\right\rangle=0, \quad \text { for } \alpha \in \mathcal{A}_{+} .
$$

or

$$
\alpha\left|\psi_{\theta=\frac{\pi}{4}}\right\rangle=0 .
$$

Hence

$$
\widehat{\mathcal{N}}_{\omega}^{+}=\left\{\left|\left(\begin{array}{ll}
\alpha_{11} & 0 \\
\alpha_{21} & 0
\end{array}\right) \otimes\left(\frac{1+\sigma_{3}}{2}\right)\right\rangle: \alpha_{i 1} \in \mathbb{C}\right\} \cong \mathbb{C}^{2} .
$$

The quotient space $\hat{\mathcal{A}} / \widehat{\mathcal{N}}_{\omega}^{+} \cong \mathbb{C}^{2}$ is spanned by

$$
\left\{\left|\left[\left(\begin{array}{cc}
0 & \alpha_{12} \\
0 & \alpha_{22}
\end{array}\right) \otimes\left(\frac{1+\sigma_{3}}{2}\right)\right]\right\rangle: \alpha_{i 2} \in \mathbb{C}\right\} .
$$

It transforms irreducibly under $\mathcal{A}^{+}$. So $\rho_{\omega}$ remains pure with zero entropy.

Case 2: $\mathcal{A}_{0}=\mathcal{A}_{-}$.

This is similar to case 1 . The null space is

$$
\widehat{\mathcal{N}}_{\omega}^{-}=\left\{\left|\left(\begin{array}{cc}
0 & \alpha_{12} \\
0 & \alpha_{22}
\end{array}\right) \otimes\left(\frac{1-\sigma_{3}}{2}\right)\right\rangle: \alpha_{i 2} \in \mathbb{C}\right\} \cong \mathbb{C}^{2},
$$

the quotient space being

$$
\hat{\mathcal{A}}^{-} / \widehat{\mathcal{N}}_{\omega}^{-}=\left\{\left|\left[\left(\begin{array}{ll}
\alpha_{11} & 0 \\
\alpha_{21} & 0
\end{array}\right) \otimes\left(\frac{1-\sigma_{3}}{2}\right)\right]\right\rangle: \alpha_{i 1} \in \mathbb{C}\right\} \cong \mathbb{C}^{2} .
$$

It transforms irreducibly under $\mathcal{A}^{-}$. So $\rho_{\omega}$ remains pure with zero entropy.

Case 3: $\mathcal{A}_{0}=\mathcal{A}_{+} \oplus \mathcal{A}_{-}$

The null space $\widehat{\mathcal{N}}_{\omega}$ is the direct sum

$$
\widehat{\mathcal{N}}_{\omega}=\widehat{\mathcal{N}}_{\omega}^{+} \oplus \widehat{\mathcal{N}}_{\omega}^{-}
$$


while the quotient space is

$$
\left(\hat{\mathcal{A}}_{+} \oplus \hat{\mathcal{A}}_{-}\right) / \widehat{\mathcal{N}}_{\omega}=\left\{\left|\left[\left(\begin{array}{ll}
0 & \alpha_{12} \\
0 & \alpha_{22}
\end{array}\right) \otimes\left(\frac{1+\sigma_{3}}{2}\right)+\left(\begin{array}{cc}
\alpha_{11} & 0 \\
\alpha_{21} & 0
\end{array}\right) \otimes\left(\frac{1-\sigma_{3}}{2}\right)\right]\right\rangle\right\}
$$

This representation is the direct sum of two irreducible representations given by the two terms in (IV.44). We must now restrict

$$
\left|\mathbb{1}_{\mathcal{A}}\right\rangle=\left(\begin{array}{ll}
1 & 0 \\
0 & 1
\end{array}\right) \otimes\left(\begin{array}{ll}
1 & 0 \\
0 & 1
\end{array}\right)
$$

into its components in $\left(\hat{\mathcal{A}}_{+} \oplus \hat{\mathcal{A}}_{-}\right) / \widehat{\mathcal{N}}_{\omega}$.

Its component in $\hat{\mathcal{A}}_{+} / \widehat{\mathcal{N}}_{\omega}^{+}$is

$$
\left(\begin{array}{ll}
0 & 0 \\
0 & 1
\end{array}\right) \otimes\left(\begin{array}{ll}
1 & 0 \\
0 & 0
\end{array}\right):=\mathbb{1}^{+}
$$

while that in $\hat{\mathcal{A}}_{-} / \widehat{\mathcal{N}}_{\omega}^{-}$is

$$
\left(\begin{array}{ll}
1 & 0 \\
0 & 0
\end{array}\right) \otimes\left(\begin{array}{ll}
0 & 0 \\
0 & 1
\end{array}\right):=\mathbb{1}^{-}
$$

The squared norm of $\left|\left[\mathbb{1}^{ \pm}\right]\right\rangle$is $1 / 2$ :

$$
\left\langle\left[\mathbb{1}^{ \pm}\right] \mid\left[\mathbb{1}^{ \pm}\right]\right\rangle=\operatorname{Tr}\left(\rho_{\omega}\left(\mathbb{1}^{ \pm}\right)^{*} \mathbb{1}^{ \pm}\right)=\frac{1}{2} .
$$

Hence

$$
\left.\rho_{\omega}\right|_{\left(\mathcal{A}_{+} \oplus \mathcal{A}_{-}\right)}=\frac{1}{2}\left(\sqrt{2}\left|\left[\mathbb{1}^{+}\right]\right\rangle\left\langle\left[\mathbb{1}^{+}\right]|\sqrt{2}+\sqrt{2}|\left[\mathbb{1}^{-}\right]\right\rangle\left\langle\left[\mathbb{1}^{-}\right]\right| \sqrt{2}\right),
$$

giving $S\left(\rho_{\omega} \mid\left(\mathcal{A}_{+} \oplus \mathcal{A}_{-}\right)\right)=\log 2$ for the entropy.

\section{Role of Hopf algebras}

In elementary quantum physics, one starts with a Hilbert space $\mathcal{H}_{A_{i}}$ which typically carries the representation of the algebra $\mathcal{A}_{A_{i}}$ of single-particle observables for particle $A_{i}$. In the second quantized version, there is an isomorphism of $\mathcal{A}_{A_{i}}$ into the full algebra $\mathcal{A}_{A_{1}, A_{2}, \ldots, A_{k}}$ of observables on

$$
\mathcal{H}_{A_{1}, A_{2}, \ldots, A_{k}}=\mathcal{H}_{A_{1}} \otimes \cdots \otimes \mathcal{H}_{A_{k}}
$$

if the particles $A_{1}, A_{2}, \ldots, A_{k}$ are non-identical. 
We must identify the $A_{1}$-particle observables in the $k$-particle Hilbert space to be able to observe properties of $A_{1}$ say in $\mathcal{H}_{A_{1}, A_{2}, \ldots, A_{k}}$. They are given by the isomorphism $\Delta^{k}$ for non-identical particles defined by

$$
\Delta^{k}\left(\alpha_{A_{1}}\right)=\alpha_{A_{1}} \otimes \mathbb{1}_{A_{2}} \otimes \cdots \otimes \mathbb{1}_{A_{k}}, \quad \alpha_{A_{1}} \in \mathcal{A}_{A_{1}}
$$

as we saw earlier.

When the particles are identical so that $A_{1}=A_{2}=\cdots=A_{k}$ and are fermions or bosons, such an isomorphism still exists: they are the totally symmetrized versions of (IV.51) as we also saw (cf. (III.14)). This is, in fact, the simplest choice of a coproduct

$$
\Delta \equiv \Delta^{1}
$$

given by

$$
\Delta^{k}(\alpha):=\alpha \otimes \mathbb{1} \otimes \cdots \otimes \mathbb{1}+\mathbb{1} \otimes \alpha \otimes \cdots \otimes \mathbb{1}+\cdots+\mathbb{1} \otimes \cdots \otimes \alpha .
$$

When the particles are identical, but fulfill braid group statistics, there still exists $\Delta^{k}$ as we will show below. As a special case, the same holds for parastatistics, its expression then is the same as for bosons and fermions.

The importance of $\Delta^{k}$ is as follows: it identifies the single particle observables in the $k$-particle Hilbert space.

The choice of $\Delta^{k}$ typically comes from the statistics group. It can differ if the latter differs. If the statistics group is the braid group or its quotient, the permutation group, then $\left(\mathcal{A}_{A_{i}}, \Delta^{k}\right)$ defines a "quasi-triangular Hopf algebra" [17]. The isomorphism $\Delta^{1} \equiv \Delta$ then defines a "coproduct" from which $\Delta^{k}$ can be deduced.

More general possibilities than braid group and accordingly more general single-particle algebras than the above can also be contemplated [24 26].

There is conceptually no problem in restricting a state $\omega$ on $\mathcal{A}_{A_{1}, A_{2}, \ldots, A_{k}}$ to the subalgebra $\Delta^{k}\left(\mathcal{A}_{A_{i}}\right)$ and comparing the entropies of $\left.\omega\right|_{\mathcal{A}_{A_{1}, A_{2}, \ldots, A_{k}}}$ and $\left.\omega\right|_{\Delta^{k}\left(\mathcal{A}_{A_{i}}\right)}$.

We may also wish to study a group of $k$-particles with an algebra $\mathcal{A}^{(k)}$ in an $n$-particle Hilbert space, for any $n>k$. If $\mathcal{A}^{(k)}$ is Hopf, then we can find its isomorphic algebra at the $n$-particle level, often more than one. The ambiguity in its choice has to be resolved by the context. Entropy considerations can again be pushed through. 


\section{Identical Particles}

In this section we illustrate the use of the GNS construction for the evaluation of entanglement entropy in systems of identical particles, making use of the coproduct in order to identify subalgebras of one-particle observables. The general setting for the three examples we consider below is the following. We consider a one-particle Hilbert space $\mathcal{H}^{(1)} \cong \mathbb{C}^{d}$. In this case, the full one-particle observable algebra is given by the group algebra of $U(d)$, $\mathbb{C} U(d)$. The two-particle Hilbert space is then given by the subspace of $\mathcal{H}^{(1)} \otimes \mathcal{H}^{(1)}$ consisting of either symmetric (bosonic statistics) or antisymmetric (fermionic statistics) tensors. The coproduct is a homomorphism $\Delta: \mathbb{C} U(d) \rightarrow \mathbb{C} U(d) \otimes \mathbb{C} U(d)$ that allows us to map one-particle observables from the one-particle sector to the two-particle sector. The map $\Delta$ is not fixed a priori. Here we consider the standard choice

$$
\Delta(g)=g \otimes g
$$

for $g \in U(d)$, linearly extended to all of $\mathbb{C} U(d)$. This coproduct is the exponentiated form of IV.53 (see also below). The crucial property is coassociativity:

$$
(\Delta \otimes \mathrm{id}) \Delta=(\mathrm{id} \otimes \Delta) \Delta
$$

This property allows us, starting from $\mathbb{C} U(d)$ and via the coproduct, to construct observables acting at the $k$-particle level, for any $k$. In the next section, other choices of the coproduct will be used in order to apply our ideas to examples with braid group statistics.

We always consider algebras $\mathcal{A}$ with unity and subalgebras $\mathcal{A}_{0}$ which contain this unity. The physical reason for including the unity of $\mathcal{A}$ in $\mathcal{A}_{0}$ will become apparent in section VI.

Now, if we perform measurements where only a restricted set of one-particle observables is considered, we may study the entanglement of a given two-particle state $|\psi\rangle$ that arises from the corresponding restriction. For example, if from the $d$ available "levels" in $\mathcal{H}^{(1)}=\mathbb{C}^{d}$ we consider only $d^{\prime}$ of them $\left(d^{\prime}<d\right)$, the algebra of one-particle observables will be reduced to the algebra generated by $\mathbb{C} U\left(d^{\prime}\right)$ and unity. Its dimension is $d^{\prime 2}+1$. The two-particle Hilbert space will then decompose into irreducible representations of this algebra, this being directly reflected in the entanglement structure of the state $|\psi\rangle$. 
1. Two Fermions, $\mathcal{H}^{(1)}=\mathbb{C}^{4}$

As a first example, we consider the case $d=4, d^{\prime}=2$. The two-fermion space $\Lambda^{2} \mathcal{H}^{(1)}$ is 6-dimensional, as can be seen from the decomposition $4 \otimes 4=10 \oplus 6$ of $\mathbb{C}^{4} \otimes \mathbb{C}^{4}$ into symmetric and antisymmetric tensors. Let us consider a particular orthonormal basis $\left\{\left|e_{1}\right\rangle,\left|e_{2}\right\rangle,\left|e_{3}\right\rangle,\left|e_{4}\right\rangle\right\}$ for $\mathcal{H}^{(1)}$. A basis for the two-fermion space $\Lambda^{2} \mathcal{H}^{(1)} \cong \mathbb{C}^{6}$ is then given by $\left\{\left|e_{i}\right\rangle \wedge\left|e_{j}\right\rangle\right\}_{1 \leq i<j \leq 4}$.

Now we will assume that only one-particle observables containing $\mathbb{1}_{4}$ and those causing transitions between the states $\left|e_{1}\right\rangle$ and $\left|e_{2}\right\rangle$ are considered. Then, the relevant algebra of observables is isomorphic to $\mathbb{C} U(2) \otimes \mathbb{1}_{4}$. Using the coproduct, we can obtain the image of this algebra acting on the two-particle sector. For our choice of the subalgebra, the relevant observables are generated by operators of the form $M_{i j}=\left|e_{i}\right\rangle\left\langle e_{j}\right|$, with $1 \leq i, j \leq 2$ and $\mathbb{1}_{4}$. In this context, it turns out to be useful to work with the "infinitesimal" version of (IV.54), that is, if $L$ is an element of the Lie algebra of $U(d)$, we set

$$
\Delta(L)=L \otimes \mathbb{1}+\mathbb{1} \otimes L
$$

It is also convenient to label the basis vectors of $\Lambda^{2} \mathcal{H}^{(1)}$ in the following way:

$$
\begin{gathered}
|a\rangle=\left|e_{1}\right\rangle \wedge\left|e_{2}\right\rangle \equiv \frac{1}{\sqrt{2}}\left(\left|e_{1}\right\rangle \otimes\left|e_{2}\right\rangle-\left|e_{2}\right\rangle \otimes\left|e_{1}\right\rangle\right), \\
\left|\alpha_{1}\right\rangle=\left|e_{1}\right\rangle \wedge\left|e_{3}\right\rangle, \quad\left|\alpha_{2}\right\rangle=\left|e_{2}\right\rangle \wedge\left|e_{3}\right\rangle \\
\left|\beta_{1}\right\rangle=\left|e_{1}\right\rangle \wedge\left|e_{4}\right\rangle, \quad\left|\beta_{2}\right\rangle=\left|e_{2}\right\rangle \wedge\left|e_{4}\right\rangle \\
|b\rangle=\left|e_{3}\right\rangle \wedge\left|e_{4}\right\rangle
\end{gathered}
$$

From IV.56) it is easy to obtain explicit expressions for the matrix representations of the relevant one-particle observables. As an illustration, we compute:

$$
\begin{aligned}
\Delta\left(M_{12}\right)\left|\alpha_{2}\right\rangle & =\Delta\left(M_{12}\right)\left|e_{2}\right\rangle \wedge\left|e_{3}\right\rangle \\
& =\left(M_{12} \otimes \mathbb{1}+\mathbb{1} \otimes M_{12}\right) \frac{1}{\sqrt{2}}\left(\left|e_{2}\right\rangle \otimes\left|e_{3}\right\rangle-\left|e_{3}\right\rangle \otimes\left|e_{2}\right\rangle\right) \\
& =\frac{1}{\sqrt{2}}\left(\left|e_{1}\right\rangle \otimes\left|e_{3}\right\rangle-\left|e_{3}\right\rangle \otimes\left|e_{1}\right\rangle\right) \\
& =\left|\alpha_{1}\right\rangle .
\end{aligned}
$$

The four matrices $A_{i j} \equiv \Delta\left(M_{i j}\right)$ (for $\left.i, j=1,2\right)$ turn out to be block diagonal in the chosen 
basis:

$$
\begin{aligned}
& A_{11}=\operatorname{diag}\left\{1, e_{11}, e_{11}, 0\right\}, \\
& A_{22}=\operatorname{diag}\left\{1, e_{22}, e_{22}, 0\right\}, \\
& A_{12}=\operatorname{diag}\left\{0, e_{12}, e_{12}, 0\right\}, \\
& A_{21}=\operatorname{diag}\left\{0, e_{21}, e_{21}, 0\right\},
\end{aligned}
$$

where $e_{i j}$ denote the standard matrix units on $M_{2}(\mathbb{C})$, i.e., $e_{11}=\left(\begin{array}{ll}1 & 0 \\ 0 & 0\end{array}\right)$, and so on. To this must be added the unit matrix $\mathbb{1}_{6}$.

The algebra $\mathcal{A}_{0}$ will be generated by exponentials of these matrices and their products. It has a basis consisting of the five matrices $A_{11}, A_{22}, A_{12}, A_{21}$ and $\mathbb{1}_{6}$. This same example can be worked using creation/annihilation operators. In that case, as explained in [2], the algebra corresponding to observables involving only the identity and transitions between $\left|e_{1}\right\rangle$ and $\left|e_{2}\right\rangle$ is six dimensional. However, one of the basis elements corresponds to a 2particle observable, being the product of the number operators of particles 1 and 2 . The 1-particle observable algebra is therefore the one obtained after taking the quotient by the ideal generated by that basis element. Here we are using coproducts in such and only 1-particle observables appear upon application of the homomorphism $\Delta$.

We now consider a $\theta$-dependent state vector, given by

$$
\left|\psi_{\theta}\right\rangle=\cos \theta\left|\beta_{1}\right\rangle+\sin \theta\left|\alpha_{2}\right\rangle
$$

As mentioned above, at the two-particle level the full observable algebra $\mathcal{A}$ given by $M_{6}(\mathbb{C})$. The subalgebra of one-particles we have chosen is the $\mathcal{A}_{0}$ constructed above. We proceed to the construction of the GNS representation corresponding to different values of $\theta$ when the state $\left|\psi_{\theta}\right\rangle$ is restricted to $\mathcal{A}_{0}$.

Case 1: $0<\theta<\frac{\pi}{2}$

When $0<\theta<\pi / 2$ we can easily check that the only non-zero elements $\alpha \in \mathcal{A}_{0}$ for which

$$
\omega_{\theta}\left(\alpha^{*} \alpha\right) \equiv\left\langle\psi_{\theta}\left|\alpha^{*} \alpha\right| \psi_{\theta}\right\rangle=0
$$

are linear combinations of $B=\operatorname{diag}\left\{1,0_{2}, 0_{2}, 0\right\}$ and $\mathbb{1}_{6}-A_{11}-A_{22}$, that is the null space $\mathcal{N}_{\theta}$ is generated by these elements. The GNS Hilbert space $\mathcal{H}_{\theta}$ is thus four-dimensional.

Let $\pi_{\theta}$ denote the corresponding GNS representation of $\mathcal{A}_{0}$ on $\mathcal{H}_{\theta}$. A convenient basis for $\mathcal{H}_{\theta}$ is given by $\left\{\left|\left[A_{i j}\right]\right\rangle\right\}_{i, j=1,2}$. A straightforward computation shows that the subspace 
spanned by $\left|\left[A_{12}\right]\right\rangle$ and $\left|\left[A_{22}\right]\right\rangle$, as well as the subspace spanned by $\left|\left[A_{11}\right]\right\rangle$ and $\left|\left[A_{21}\right]\right\rangle$, are irreducible. The two representations are isomorphic.

The corresponding projections $P_{1}$ and $P_{2}$ can then be used in order to obtain the components of $\left|\left[\mathbb{1}_{6}\right]\right\rangle$ in each irreducible subspace. From

$$
\left|\left[\mathbb{1}_{6}\right]\right\rangle=\left|\left[A_{11}+A_{22}\right]\right\rangle
$$

we obtain

$$
P_{1}\left|\left[\mathbb{1}_{6}\right]\right\rangle=\left|\left[A_{11}\right]\right\rangle, \quad P_{2}\left|\left[\mathbb{1}_{6}\right]\right\rangle=\left|\left[A_{22}\right]\right\rangle .
$$

Using (IV.63), we compute

$$
\| P_{1}\left|\left[\mathbb{1}_{6}\right]\right\rangle\left\|^{2}=\cos ^{2} \theta, \quad\right\| P_{2}\left|\left[\mathbb{1}_{6}\right]\right\rangle \|^{2}=\sin ^{2} \theta
$$

A density matrix acting on the GNS space of the restricted state can be obtained as explained above and its entropy can be computed. The result is:

$$
S(\theta)=-\cos ^{2} \theta \log \cos ^{2} \theta-\sin ^{2} \theta \log \sin ^{2} \theta
$$

Case 2: $\theta=0$.

In this case we have

$$
\left|\psi_{0}\right\rangle=\left|\beta_{1}\right\rangle
$$

So, the space of null vectors is given by the four-dimensional space

$$
\mathcal{N}_{0,0}=\operatorname{Span}\left\{|B\rangle,\left|\left[\mathbb{1}_{6}-A_{11}\right]\right\rangle,\left|\left[A_{22}\right]\right\rangle,\left|\left[A_{12}\right]\right\rangle\right\}
$$

This means that $\mathcal{H}_{\theta=0} \cong \mathbb{C}^{2}$. Hence, the representation is irreducible so that the corresponding entropy vanishes.

The situation is completely equivalent for the case $\theta=\frac{\pi}{2}$.

Thus, $\mathcal{H}_{\theta}$ decomposes into irreducible subspaces according to the following pattern:

$$
\mathcal{H}_{\theta} \cong\left\{\begin{array}{cc}
\mathbb{C}^{2}, & \theta=0, \pi / 2 \\
\mathbb{C}^{4} \cong \mathbb{C}^{2} \oplus \mathbb{C}^{2}, & \theta \in(0, \pi / 2) .
\end{array}\right.
$$

The significant aspect of this example is the fact that for the values of $\theta$ for which the Slater rank of $\left|\psi_{\theta}\right\rangle$ is one, namely $\theta=0$ and $\frac{\pi}{2}$, we obtain exactly zero for the entropy. In previous treatments of entanglement for identical particles, the minimum value for the von Neumann entropy of the reduced density matrix (obtained by partial trace) has been found to be $\log 2$. 
This has been a source of embarrassment: it seems to suggest that different entanglement criteria have to be adopted, depending on whether one is dealing with non-identical particles, or with bosons, or fermions. For a critical review of previous attempts at a solution to this problem, see [27]. We have shown here that, by replacing the notion of partial trace by the more general one of restriction to a subalgebra, all cases can be treated on an equal footing.

\section{Two Fermions, $\mathcal{H}^{(1)}=\mathbb{C}^{3}$}

As a second example, we consider the case $d=3$, with two choices for $d^{\prime}: d^{\prime}=2,3$. In this case, the two-fermion space is given by $\mathcal{H}^{(2)}=\Lambda^{2} \mathbb{C}^{3} \subset \mathcal{H}^{(1)} \otimes \mathcal{H}^{(1)}$. Let $\left\{\left|e_{1}\right\rangle,\left|e_{2}\right\rangle,\left|e_{3}\right\rangle\right\}$ denote an orthonormal basis for $\mathcal{H}^{(1)}$. Then

$$
\left\{\left|f^{k}\right\rangle:=\varepsilon^{i j k}\left|e_{i} \wedge e_{j}\right\rangle\right\}_{1 \leq k \leq 3},
$$

provides an orthonormal basis for $\mathcal{H}^{(2)}$. This choice of basis is particularly useful if we take into account that it provides a basis for the $(S U(3))$ representation $\overline{3}$ obtained from the decomposition $3 \otimes 3=6 \oplus \overline{3}$, corresponding to symmetrisation/antisymmetrisation of tensors in $\mathcal{H}^{(1)} \otimes \mathcal{H}^{(1)}$. The $\left|f^{i}\right\rangle$ span the $\overline{3}$ representation.

Here, the representation 3 stands for the defining $U(3)$ representation on $\mathcal{H}^{(1)}\left(U^{(1)}(g)=\right.$ $g$ ). This means that, at the two fermion level, one particle observables are given by the action of $\mathbb{C} U(3)$ on $\mathcal{H}^{(2)}$. This action is obtained from the restriction of operators of the form $\widehat{\alpha}=\int_{U(d)} d \mu(g) \alpha(g) U^{(1)}(g) \otimes U^{(1)}(g)$ to $\overline{3}$, regarded as a subspace of $3 \times 3$.

It follows that the algebra $\mathcal{A}$ of observables for the two-fermion system is generated by $\left|f^{i}\right\rangle\left\langle f^{j}\right|(i, j=1,2,3)$. Hence, $\mathcal{A} \cong M_{3}(\mathbb{C})$.

Below we consider two different choices for the subalgebra $\mathcal{A}_{0}$. This will make clear that the notion of entanglement is very sensitive not only to the choice of state, but also to the choice of the observable algebra.

Choice 1 for $\mathcal{A}_{0}$ :

Here we consider $\mathcal{A}_{0}$ to be the full algebra $\mathcal{A}$. That is, $\mathcal{A}_{0}$ is chosen to be the full algebra of one-particle observables acting on $\mathcal{H}^{(2)}$. Now, we pick any two-fermion pure state $\omega: \mathcal{A} \rightarrow \mathbb{C}$. Being a pure state on $\mathcal{A} \cong A_{0}$, the GNS representation corresponding to the pair $\left(\mathcal{A}_{0}, \omega\right)$ is irreducible. This is equivalent to the statement that $\overline{3}$ is an irreducible $S U(3)$ representation. This in turn corresponds to the well-known fact that, for $\mathcal{H}^{(1)}=\mathbb{C}^{3}$, 
all two-fermion vector states have Slater rank 1 (cf. [8]). These states are therefore to be considered as non-entangled states. Notice, however, that if we use partial trace to compute the von Neumann entropy we get a result different from zero. In contrast, computing the von Neumann entropy via the GNS construction gives automatically zero in this case (because of irreducibility), this being in accordance with the fact that all states for $d=3$ are nonentangled (as long as $\mathcal{A}_{0}=\mathcal{A}$ ).

Choice 2 for $\mathcal{A}_{0}$ :

Now we let $\mathcal{A}_{0}$ be the subalgebra of $\mathcal{A}$ consisting of all one-particle observables that involve only the one-particle states $\left|e_{1}\right\rangle$ and $\left|e_{2}\right\rangle$ and unity $\mathbb{1}_{\mathcal{A}}=\mathbb{1}_{3}$. It can be easily checked that this subalgebra is generated by the operators $M^{i j}:=\left|f^{i}\right\rangle\left\langle f^{j}\right|(i, j=1,2)$, as well as $\mathbb{1}_{\mathcal{A}}$. This is, therefore, a five dimensional matrix algebra.

Consider the following two-fermion vector state:

$$
\left|\psi_{\theta}\right\rangle=\cos \theta\left|f^{1}\right\rangle+\sin \theta\left|f^{3}\right\rangle
$$

and let $\omega_{\theta}: \mathcal{A} \rightarrow \mathbb{C}$ denote the corresponding state:

$$
\omega_{\theta}(\alpha)=\left\langle\psi_{\theta}|\alpha| \psi_{\theta}\right\rangle, \quad \forall \alpha \in \mathcal{A}
$$

Consider now the restriction of $\omega_{\theta}$ to the subalgebra $\mathcal{A}_{0}$ :

$$
\omega_{\theta, 0}=\left.\omega_{\theta}\right|_{\mathcal{A}_{0}} .
$$

We proceed to perform the GNS construction corresponding to the pair $\mathcal{A}_{0}, \omega_{\theta, 0}$, assuming that $0<\theta<\frac{\pi}{2}$. By direct computation we check that both $M^{12}$ and $M^{22}$ are null vectors:

$$
\left|\left[M^{12}\right]\right\rangle=\left|\left[M^{22}\right]\right\rangle=0
$$

In this range of values for $\theta$ these are all the linearly independent null vectors. This can be seen from

$$
\left\langle\psi_{\theta}\left|\alpha^{*} \alpha\right| \psi_{\theta}\right\rangle=0 \Rightarrow \alpha\left|\psi_{\theta}\right\rangle=0 \Rightarrow \alpha=\sum c_{i} M^{i 2}, c_{i} \in \mathbb{C}
$$

This means that the null space $\mathcal{N}_{\theta, 0}$ is two-dimensional and that, therefore, $\mathcal{H}_{\theta}=\hat{\mathcal{A}}_{0} / \mathcal{N}_{\theta, 0}$ is isomorphic to $\mathbb{C}^{3}$, with basis $\left\{\left|\left[M^{11}\right]\right\rangle,\left|\left[M^{21}\right]\right\rangle,\left|\left[E^{3}\right]\right\rangle\right\}$, where $E^{3}:=\mathbb{1}_{\mathcal{A}}-M^{11}-M^{22}$.

Noticing that $\alpha_{0} \in \mathcal{A}_{0}$ implies $\alpha_{0} E^{3}=0$, we can check that the GNS space has the following decomposition in terms of irreducible representations: $\mathcal{H}_{\theta}=\mathbb{C}^{2} \oplus \mathbb{C}^{1}$. 
Denoting $P_{1}$ and $P_{2}$ the corresponding projections and using the fact that $\left[M^{11}+\right.$ $\left.M^{22}\right]=\left[\mathbb{1}_{2}\right]$, we obtain

$$
P_{1}\left|\left[\mathbb{1}_{\mathcal{A}}\right]\right\rangle=\left|\left[M^{11}\right]\right\rangle, \quad P_{2}\left|\left[\mathbb{1}_{\mathcal{A}}\right]\right\rangle=\left|\left[E^{3}\right]\right\rangle
$$

Using the inner product of $\mathcal{H}_{\theta}$ to compute $\left|\mu_{i}\right|^{2}=\| P_{i}\left|\left[\mathbb{1}_{\mathcal{A}}\right]\right\rangle \|^{2}$, we obtain

$$
\left|\mu_{1}\right|^{2}=\cos ^{2} \theta, \quad\left|\mu_{2}\right|^{2}=\sin ^{2} \theta
$$

Hence,

$$
\omega_{\theta, 0}=\cos ^{2} \theta\left(\frac{1}{\cos ^{2} \theta}\left|\left[M^{11}\right]\right\rangle\left\langle\left[M^{11}\right]\right|\right)+\sin ^{2} \theta\left(\frac{1}{\sin ^{2} \theta}\left|\left[E^{3}\right]\right\rangle\left\langle\left[E^{3}\right]\right|\right) .
$$

The result for the entropy as a function of $\theta$ is therefore

$$
S(\theta)=-\cos ^{2} \theta \log \cos ^{2} \theta-\sin ^{2} \theta \log \sin ^{2} \theta
$$

The cases $\theta=0$ and $\theta=\pi / 2$ differ from the above mainly in the dimension of the GNS Hilbert space. Nevertheless, the result for the entropy remains the same. It is given by the same formula (IV.80), extended now to the values $\theta=0$ and $\theta=\pi / 2$. This means that the entropy for $\theta=0$ or $\theta=\pi / 2$ is zero, and corresponds to the fact that, for these values of $\theta$, the GNS Hilbert space is irreducible. Indeed, the result for the GNS space in the range $\theta \in[0, \pi / 2]$ is:

$$
\mathcal{H}_{\theta} \cong\left\{\begin{array}{cc}
\mathbb{C}^{2}, & \theta=0 \\
\mathbb{C}^{3} \cong \mathbb{C}^{2} \oplus \mathbb{C}, & \theta \in(0, \pi / 2) \\
\mathbb{C}, & \theta=\pi / 2 .
\end{array}\right.
$$

This result should be contrasted against the fact that the $\overline{3}$ representation, when regarded as a representation space for $S U(2)$, splits as $2 \oplus 1$.

\section{Two Bosons, $\mathcal{H}^{(1)}=\mathbb{C}^{3}$}

This is the bosonic counterpart of the previous example. Let $\left\{\left|e_{1}\right\rangle,\left|e_{2}\right\rangle,\left|e_{3}\right\rangle\right\}$ denote an orthonormal basis for $\mathcal{H}^{(1)}=\mathbb{C}^{3}$. Recalling the decomposition $3 \otimes 3=6 \oplus \overline{3}$ of the previous example, we see that the six-dimensional space corresponds to the two-particle space $\mathcal{H}^{(2)}$, i.e., the space of symmetric tensors in $\mathcal{H}^{(1)} \otimes \mathcal{H}^{(1)}$. The algebra $\mathcal{A}$ of observables for this 
two-boson system is then isomorphic to $M_{6}(\mathbb{C})$. As a basis for $\mathcal{H}^{(2)}$ we choose the vectors $\left\{\left|e_{i} \vee e_{j}\right\rangle\right\}_{i, j \in\{1,2,3\}}$, where

$$
\left|e_{i} \vee e_{j}\right\rangle \equiv\left\{\begin{array}{cc}
\frac{1}{\sqrt{2}}\left(\left|e_{i}\right\rangle \otimes\left|e_{j}\right\rangle+\left|e_{j}\right\rangle \otimes\left|e_{i}\right\rangle\right), & i \neq j \\
\left|e_{i}\right\rangle \otimes\left|e_{i}\right\rangle, & i=j
\end{array}\right.
$$

They form an orthonormal basis.

Now we consider the (pure) state $\omega_{(\theta, \phi)}: \mathcal{A} \rightarrow \mathbb{C}$ that corresponds to

$$
\left|\psi_{(\theta, \phi)}\right\rangle=\sin \theta \cos \phi\left|e_{1} \vee e_{2}\right\rangle+\sin \theta \sin \phi\left|e_{1} \vee e_{3}\right\rangle+\cos \theta\left|e_{3} \vee e_{3}\right\rangle
$$

We are interested in the restriction of $\omega_{(\theta, \phi)}$ to the subalgebra $\mathcal{A}_{0}$ of one-particle observables which besides $\mathbb{1}_{6}$, pertains only to the one-particle vectors $\left|e_{1}\right\rangle$ and $\left|e_{2}\right\rangle$. Proceeding in the same way as in the previous examples, we recognize that the 6 representation, when regarded as a representation space for $S U(2)$ acting nontrivially on $\left|e_{1}\right\rangle$ and $\left|e_{2}\right\rangle$, splits as $6=3 \oplus 2 \oplus 1$. The basis vectors for these three invariant subspaces are given below:

$$
\begin{array}{ll}
3: & |1\rangle=\left|e_{1} \vee e_{1}\right\rangle,|0\rangle=\left|e_{1} \vee e_{2}\right\rangle,|-1\rangle=\left|e_{2} \vee e_{2}\right\rangle, \\
2: & |1 / 2\rangle=\left|e_{1} \vee e_{3}\right\rangle,|-1 / 2\rangle=\left|e_{2} \vee e_{3}\right\rangle, \\
1: & |\tilde{0}\rangle=\left|e_{3} \vee e_{3}\right\rangle .
\end{array}
$$

The one-particle observables on $\mathcal{H}^{(2)}$ are obtained from the operators $\left|e_{i}\right\rangle\left\langle e_{j}\right|$ (with $i, j=$ $1,2)$, as well as from the unit operator on $\mathcal{H}^{(1)}$, by means of the coproduct. Thus, the subalgebra $\mathcal{A}_{0}$ is generated by operators of the form $|u\rangle\langle v|$, with both $|u\rangle$ and $|v\rangle$ belonging to the same irreducible component of $\mathcal{H}^{(2)}$. (Note that the image of unity on $\mathcal{H}^{(1)}$ under the coproduct $\Delta$ is $\mathbb{1}_{\mathcal{A}}$. Hence by taking combinations of images of the above $\mathcal{H}^{(1)}$-observables under $\Delta$, we see that $\mathcal{A}_{0}$ contains $\left.|\tilde{0}\rangle\langle\tilde{0}|\right)$. In other words, $\mathcal{A}_{0}$ is given by block-diagonal matrices, with each block corresponding to one of the irreducible components in the decomposition $6=3 \oplus 2 \oplus 1$. The dimension of $\mathcal{A}_{0}$ is therefore $3^{2}+2^{2}+1^{2}=14$.

The construction of the GNS-representation corresponding to each particular value of the parameters $\theta$ and $\phi$ is performed following the same procedure as in the previous examples. Let us introduce the notation $B_{u, v} \equiv|u\rangle\langle v|$, for any pair $|u\rangle,|v\rangle$ in (IV.84). Then, from (IV.83) we see that as long as the $(\theta, \phi)$-coefficients are all different from zero, those elements of $\mathcal{A}_{0}$ of the form $B_{j, \pm 1}(j=0, \pm 1)$ and $B_{\sigma,-1 / 2}(\sigma= \pm 1 / 2)$ generate the null vectors. That these generate all the null vectors follows from the fact that (IV.83) contains one basis 
element for every irreducible component, so that no further linear relation can arise that lead to null vectors. So in this case we have

$$
\mathcal{H}_{(\theta, \phi)}:=\hat{\mathcal{A}}_{0} / \mathcal{N}_{(\theta, \phi), 0}=\mathbb{C}^{6}, \quad \mathcal{N}_{(\theta, \phi), 0}=\text { Null space }
$$

In terms of irreducible subspaces, one can readily see that $\mathbb{C}^{6}$ decomposes according to $\mathbb{C}^{6}=\mathbb{C}^{3} \oplus \mathbb{C}^{2} \oplus \mathbb{C}^{1}$

In general, we can read off the decomposition of $\mathcal{H}_{(\theta, \phi)}$ into irreducible subspaces from IV.83), depending on which of its coefficents vanish. For example, if only the first one vanishes,

$$
\mathcal{H}_{(\theta, \phi)}=\mathbb{C}^{2} \oplus \mathbb{C}^{1}
$$

It is interesting to consider the entropy as a function of $(\theta, \phi)$. For the case in which all $(\theta, \phi)$-coefficients are non-zero, we have:

$$
\left|\left[\mathbb{1}_{\mathcal{A}}\right]\right\rangle=\left|\left[B_{1,1}\right]\right\rangle+\left|\left[B_{1 / 2,1 / 2}\right]\right\rangle+\left|\left[B_{\tilde{0}, \tilde{0}}\right]\right\rangle
$$

from which the entropy is readily computed as before. The result is:

$$
S(\theta, \phi)=-\sin ^{2} \theta\left[\cos ^{2} \phi \log (\sin \theta \cos \phi)^{2}+\sin ^{2} \phi \log (\sin \theta \sin \phi)^{2}\right]-\cos ^{2} \theta \log (\cos \theta)^{2} .
$$

The analytic formulae for entropy when one or more of the coefficients in (IV.84) vanish can be obtained from (IV.88) by taking suitable limits on $\theta$ and $\phi$.

We can see that the entropy vanishes whenever $\left|\psi_{(\theta, \phi)}\right\rangle$ lies in a single irreducible component. This happens precisely at those points of the two-sphere generated by the parameters $(\theta, \phi)$ that correspond to the coordinate axes. There are therefore six points where the entropy vanishes exactly. This is depicted in Figure 1, where the $(\theta, \phi)$-sphere has been mapped to the $x-y$ plane through a stereographic projection. The figure shows the entropy as a function of the coordinates of that plane.

\section{E. Entanglement for Braid Group Statistics}

Let us now outline how this approach can be used to compute entanglement entropy for systems with braid statistics. For that we need to recollect some facts from [28] of the quantum group $U_{q}(s u(n))$. 


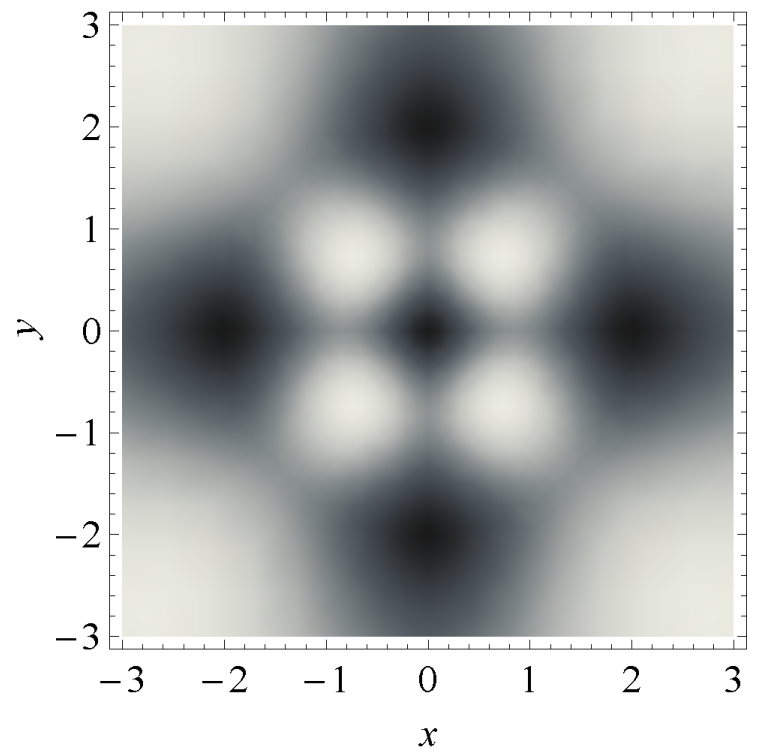

FIG. 1. The entropy equation (IV.88) as a function of $x$ and $y$, the coordinates of a plane representing the $(\theta, \phi)$-sphere through stereographic projection. Darker regions correspond to lower values of the entropy. Five of the six vanishing points of the entropy can be seen on the picture (black spots). The sixth one, corresponding to the north-pole of the sphere, lies 'at infinity' in this representation.

\section{Preliminaries: Bosonic Realization of $U_{q}(s u(n))$}

The $q$-number $[s]_{q}$ is defined by

$$
[s]_{q}=\frac{q^{\frac{s}{2}}-q^{-\frac{s}{2}}}{q^{\frac{1}{2}}-q^{-\frac{1}{2}}} .
$$

It satisfies the following properties of importance for us:

1. $[s+t]_{q}=q^{-\frac{s}{2}}[t]_{q}+q^{\frac{t}{2}}[s]_{q}$.

2. Jacobi identity: $[r]_{q}[s-t]_{q}+[s]_{q}[t-r]_{q}+[t]_{q}[r-s]_{q}=0$.

3. $[1]_{q}=1$. By definition, $[0]_{q}=0$.

Let $A, A^{\dagger}$ be $q$-deformed oscillators and let $a, a^{\dagger}$ be the standard undeformed bosonic oscillators. They are related by a dressing transformation:

$$
\begin{aligned}
A & =a \sqrt{\frac{[N]_{q}}{N}}=\sqrt{\frac{[N+1]_{q}}{N+1}} a, \\
A^{\dagger} & =\sqrt{\frac{[N]_{q}}{N}} a^{\dagger},
\end{aligned}
$$


where

$$
N=a^{\dagger} a
$$

From this dressing transformation we may find the representation for the $q$-deformed oscillators $A, A^{\dagger}$ from the known representation of $a, a^{\dagger}$. In particular, both sets have the same vacuum $|0\rangle$ such that $A|0\rangle=a|0\rangle=0$.

From (IV.90, IV.91), we see that

$$
\begin{aligned}
A^{\dagger} A & =[N]_{q}, A A^{\dagger}=[N+1]_{q}, \\
N & =a^{\dagger} a
\end{aligned}
$$

This gives

$$
A A^{\dagger}-q^{\frac{1}{2}} A^{\dagger} A=q^{-\frac{N}{2}}
$$

Also

$$
\begin{aligned}
{\left[N, A^{\dagger}\right]=A^{\dagger}, } & {[N, A]=-A ; } \\
{\left[N, a^{\dagger}\right]=a^{\dagger}, } & {[N, a]=-a . }
\end{aligned}
$$

We may now construct $U_{q}(s u(n))$ using a set of $N A_{i}, A_{i}^{\dagger}$ oscillators with a fix $q$ following the Schwinger procedure. Let $\lambda_{a}$, with $a=1, . ., n^{2}-1$, be the $n \times n$ Gell-Mann matrices of $s u(n)$. Then

$$
\Lambda_{a}=A_{i}^{\dagger}\left(\lambda_{a}\right)^{i j} A_{j}
$$

are the generators of $U_{q}(s u(n))$. We may organize this set of generators in the CartanChevalley basis, where $H_{i}$, with $i=1, . ., n-1$, generates the $U_{q}(s u(n))$ Cartan subalgebra, and $E_{ \pm \alpha}$ are ladder operators:

$$
\begin{aligned}
& E_{i j}=A_{i}^{\dagger} A_{j}, \quad E_{i j}^{\dagger}=A_{j}^{\dagger} A_{i}, \quad \text { for } i<j, \text { for } l \leq n-1, \\
& H_{l}=\frac{1}{2}\left(N_{l}-N_{l+1}\right), \quad
\end{aligned}
$$

where

$$
N_{l}=a_{l}^{\dagger} a_{l}
$$

In the case of $U_{q}(s u(2))$, the Gell-Mann matrices are the $2 \times 2$ Pauli matrices so that

$$
\Lambda_{a}=A_{i}^{\dagger}\left(\sigma_{a}\right)^{i j} A_{j}, \quad \text { with } a=1,2,3 \text { and } i=1,2 \text {. }
$$


From this we obtain

$$
\begin{gathered}
E_{12}:=J_{+}=A_{1}^{\dagger} A_{2}, \quad E_{21}:=J_{-}=A_{2}^{\dagger} A_{1}, \\
H_{1}:=J_{3}=\frac{N_{1}-N_{2}}{2},
\end{gathered}
$$

which satisfy

$$
\begin{gathered}
{\left[J_{3}, J_{ \pm}\right]= \pm J_{ \pm}} \\
{\left[J_{+}, J_{-}\right]=\left[2 J_{3}\right]_{q}}
\end{gathered}
$$

From now on, we consider only the cases for which $q$ is real and positive, $q>0$. In this case, $U_{q}(s u(n))$ is a $*$-Hopf algebra with co-product

$$
\begin{aligned}
& \Delta\left(J_{ \pm}\right)=q^{-\frac{J_{3}}{2}} \otimes J_{ \pm}+J_{ \pm} \otimes q^{\frac{J_{3}}{2}}, \\
& \Delta\left(J_{3}\right)=\mathbb{1} \otimes J_{3}+J_{3} \otimes \mathbb{1} .
\end{aligned}
$$

The unitary irreducible representations (UIRR's) of $U_{q}(s u(2))$ are labeled by $j \in \mathbb{Z}^{+} / 2$. For fixed $j,-j \leq m \leq j$, an orthonormal basis for the carrier vector space is

$$
|j m\rangle=\frac{\left(A_{1}^{\dagger}\right)^{j+m}\left(A_{2}^{\dagger}\right)^{j-m}}{\sqrt{[j+m]_{q} ![j-m]_{q} !}}|0\rangle,
$$

where for $k \in \mathbb{Z}^{+},[k]_{q} !=[1]_{q}[2]_{q} \ldots[k]_{q}$. The generators $J_{ \pm}$and $J_{3}$ act in (IV.105) as expected:

$$
\begin{aligned}
J_{ \pm}|j m\rangle & =\sqrt{[j \mp m]_{q}[j \pm m+1]_{q}}|j, m \pm 1\rangle, \\
J_{3}|j m\rangle & =m|j m\rangle .
\end{aligned}
$$

\section{The Braid Group}

We illustrate the ideas using the "two-particle" representation of $U_{q}(s u(2))$.

For the undeformed oscillators, we can easily see that the vector state $a_{1}^{\dagger}|0\rangle \otimes a_{2}^{\dagger}|0\rangle$ for example decomposes in terms of the action of the symmetric group $\mathcal{S}_{2}$ as

$$
\begin{aligned}
a_{1}^{\dagger}|0\rangle \otimes a_{2}^{\dagger}|0\rangle & =\frac{1}{2}\left[\left(a_{1}^{\dagger}|0\rangle \otimes a_{2}^{\dagger}|0\rangle+a_{2}^{\dagger}|0\rangle \otimes a_{1}^{\dagger}|0\rangle\right)+\left(a_{1}^{\dagger}|0\rangle \otimes a_{2}^{\dagger}|0\rangle-a_{2}^{\dagger}|0\rangle \otimes a_{1}^{\dagger}|0\rangle\right)\right], \\
& \equiv|1,2\rangle_{S}+|1,2\rangle_{A},
\end{aligned}
$$

where indexes $S, A$ stand for symmetric and anti-symmetric, respectively. 
For the $q$-deformed case, thi symmetric group decomposition has to be changed. This is simple to see.

We start with the highest weight state, call it $|1,1\rangle$, with respect to $U_{q}(s u(2))$ :

$$
\begin{aligned}
|1,1\rangle & =A_{1}^{\dagger}|0\rangle \otimes A_{1}^{\dagger}|0\rangle \\
\Delta\left(J_{+}\right)|1,1\rangle & =0 \\
\Delta\left(J_{3}\right)|1,1\rangle & =|1,1\rangle .
\end{aligned}
$$

Next we lower using $J_{-}$:

$$
\begin{aligned}
\Delta\left(J_{-}\right)|1,1\rangle & =\left[q^{-\frac{J_{3}}{2}} \otimes J_{-}+J_{-} \otimes q^{\frac{J_{3}}{2}}\right] A_{1}^{\dagger}|0\rangle \otimes A_{1}^{\dagger}|0\rangle \\
& =\left[q^{-\frac{1}{2}} A_{1}^{\dagger}|0\rangle \otimes A_{2}^{\dagger}|0\rangle+q^{\frac{1}{2}} A_{2}^{\dagger}|0\rangle \otimes A_{1}^{\dagger}|0\rangle\right] .
\end{aligned}
$$

It is thus clear that this state vector cannot be decomposed under the symmetric group, since the $q$-powers are breaking the structure of the symmetric tensor product.

Formally, the structure we have just seen carries a representation of the braid group. We will not go into details here which can be found in [17, 28]. In particular, as discussed by Biedenharn and Lohe, the multiparticle $q$-boson states constructed from the $q$-deformed oscillators are invariant under the braid group.

\section{Example}

The Schwinger realization of $q$-deformed oscillators given above is adapted to treat bosons. So we generalize the $q=1$ bosonic example above where $\mathcal{H}=\mathbb{C}^{3}$ and we observe just the algebra generated by the observables mixing 1 and 2 and the unit operator.

Thus we now have three $q$-deformed oscillators $A_{i}, A_{i}^{\dagger}$ which commute for $i \neq j$. The two-

particle $q$-boson states are spanned by $A_{i}^{\dagger} A_{j}^{\dagger}|0\rangle$ and is six-dimensional. Following (IV.84), we construct the orthonormal basis for the 3 subspaces invariant under the observables acting on 1 and 2 particles and the unit operator:

$$
\begin{aligned}
& 3:|1\rangle_{q}=\frac{1}{\sqrt{[2]_{q}}}\left(A_{1}^{\dagger}\right)^{2}|0\rangle, \quad|0\rangle_{q}=A_{1}^{\dagger} A_{2}^{\dagger}|0\rangle, \quad|-1\rangle_{q}=\frac{1}{\sqrt{[2]_{q}}}\left(A_{2}^{\dagger}\right)^{2}|0\rangle, \\
& 2:|1 / 2\rangle_{q}=A_{1}^{\dagger} A_{3}^{\dagger}|0\rangle, \quad|-1 / 2\rangle_{q}=A_{2}^{\dagger} A_{3}^{\dagger}|0\rangle, \\
& 1: \quad|\tilde{0}\rangle_{q}=\frac{1}{\sqrt{[2]_{q}}}\left(A_{3}^{\dagger}\right)^{2}|0\rangle .
\end{aligned}
$$


We also generalize the vector state $(\mathrm{IV} .83)$ to the normalized vector state

$$
\left|\psi_{(\theta, \phi)}\right\rangle_{q}=\left(\sin \theta \cos \phi A_{1}^{\dagger} A_{2}^{\dagger}+\sin \theta \sin \phi A_{1}^{\dagger} A_{3}^{\dagger}+\cos \theta \frac{1}{\sqrt{[2]_{q}}}\left(A_{3}^{\dagger}\right)^{2}\right)|0\rangle .
$$

It induces a state $\omega_{(\theta, \phi)}^{(q)}$ on the full algebra of $6 \times 6$ matrices.

We now restrict $\omega_{(\theta, \phi)}^{(q)}$ to the observables pertaining to operators acting on 1 and 2 indices and the unit operator. This subalgebra $\mathcal{A}_{0}(q)$ is spanned by

$$
|i\rangle_{q q}\langle j|, \quad i, j \in[-1,0,1], \quad \text { and } \quad \mathbb{1}_{6} .
$$

Now, the algebra $\mathcal{A}_{0}(q)$ generated by $\mathrm{IV} .115$ and the scalar products induced by $\omega_{(\theta, \phi)}^{(q)}$ are all independent of $q$. The conclusion is that the GNS Hilbert space and its properties are all quite independent of $q$. That includes entropy as well.

These observations can be generalized to more involved situations.

This simple example, to be contrasted with the usual two-fermion system already worked out here, shows once more that our approach allows one to naturally obtain a zero von Neumann entropy for separable systems even in the case of more sophisticated statistics. We think that this sets the stage for a more comprehensive study of systems with braid statistics, like Kitaev model, that may play a crucial role for developments of quantum computation.

\section{TIME EVOLUTION}

If a unitary time evolution $U(t)$ of a pure state $\omega$ on an algebra $\mathcal{A}$ is given, then the time evolution of its restriction $\left.\omega\right|_{\mathcal{A}_{0}}=\omega_{0}$ is determined by

$$
\omega_{0} \rightarrow \omega_{0}(t)=\left.[U(t) \omega]\right|_{\mathcal{A}_{0}}, \quad \omega_{0}(0)=\omega_{0}
$$

The evolution of $\omega_{0}$ is in general by positive maps. This fact is a consequence of the Stinespring-Choi theorem. That is the case even when $U(t)$ gives a unitary evolution on $\omega$ with Hamiltonian $H$ :

$$
U(t) \omega=e^{i H t} \omega e^{-i H t}
$$

Here $\omega$ is a density matrix.

An important point is that the rank of $\omega_{0}(t)$ need not be continuous in $t$ even if that of $U(t) \omega$ is continuous in $t$. It can change discontinuously. This is shown by the example 
below. In that example, entropy is periodic in time, not monotone increasing in time, as is thought to be the case in nature.

The case of a fermion with 3 internal degrees of freedom treated in section IVD is a simple example. The single particle Hilbert space $\mathcal{H}^{(1)}$ was $\mathbb{C}^{3}$ with orthonormal basis $\left\{\left|e_{i}\right\rangle\right\}_{i=1,2,3}$. The two-particle state space was $\overline{3}=\bigwedge^{2} \mathcal{H}^{(1)} \equiv \mathcal{H}^{(2)}$, with an orthonormal basis $\left\{\left|f^{i}\right\rangle=\varepsilon^{i j k}\left|e_{j} \wedge e_{k}\right\rangle\right\}_{i=1,2,3}$.

The single particle algebra acting on $\mathcal{H}^{(2)}$ was $\mathbb{C} U(3) \otimes \mathbb{1}_{6}$. We chose the pure state

$$
\omega_{\theta}=\left|\psi_{\theta}\right\rangle\left\langle\psi_{\theta}|, \quad| \psi_{\theta}\right\rangle=\cos \theta\left|f^{1}\right\rangle+\sin \theta\left|f^{2}\right\rangle
$$

in the two-particle sector.

The subalgebra $\mathcal{A}_{0}$ was the image under the coproduct of the single particle algebra on $\mathcal{H}^{(1)}$ acting just on $\left|e_{1}\right\rangle$ and $\left|e_{2}\right\rangle$.

Our results pertinent for the discussion of time evolution were

$$
\omega_{\theta, 0}=\cos ^{2} \theta \rho_{\theta}^{(1)}+\sin ^{2} \theta \rho_{\theta}^{(3)}
$$

where

$$
\begin{aligned}
& \rho_{\theta}^{(1)}=\frac{1}{\cos ^{2} \theta}\left|\left[M^{11}\right]\right\rangle\left\langle\left[M^{11}\right]\right|, \\
& \rho_{\theta}^{(3)}=\frac{1}{\sin ^{2} \theta}\left|\left[E^{3}\right]\right\rangle\left\langle\left[M^{3}\right]\right|
\end{aligned}
$$

and

$$
\mathcal{H}_{\theta}^{\mathrm{GNS}}=\left\{\begin{array}{cc}
\mathbb{C}^{2}, & \theta=0, \\
\mathbb{C}^{3}=\mathbb{C}^{2} \oplus \mathbb{C}, & 0<\theta<\pi / 2, \\
\mathbb{C}, & \theta=\pi / 2 .
\end{array}\right.
$$

Thus the rank of $\omega_{\theta, 0}=\left.\omega_{\theta}\right|_{\mathcal{A}_{0}}$ jumps from 2 to 1 as $\theta$ approaches 0 or $\pi / 2$.

Now consider the unitary evolution of $\left|\psi_{\theta}\right\rangle$ and $\omega_{\theta}$ under the self-adjoint Hamiltonian

$$
H=-i\left|f^{2}\right\rangle\left\langle f^{1}|+i| f^{1}\right\rangle\left\langle f^{2}\right|
$$

It generates rotations in the plane of $\left\{\left|f^{1}\right\rangle,\left|f^{2}\right\rangle\right\}$ and hence changes $\theta$ :

$$
e^{i t H}\left|\psi_{\theta}\right\rangle=\cos (\theta+t)\left|f^{1}\right\rangle+\sin (\theta+t)\left|f^{2}\right\rangle
$$

The restriction of this evolution to $\omega_{\theta, 0}$ is

$$
U(t): \omega_{\theta, 0} \rightarrow \omega_{\theta+t, 0}
$$


It is not unitary. It does not even preserve the rank of $\omega_{\theta, 0}$ : it jumps from 2 to 1 and back as $t$ increases.

We can write time evolution as positive maps so long as the rank of the density matrix stays constant or decreases. Thus consider $\omega_{\theta, 0}$ for $0<\theta<\pi / 2$. It is of rank 2 expressible in terms of the orthonormal eigenvectors

$$
\left|\chi^{(1)}(\theta)\right\rangle=\frac{1}{\cos \theta}\left|\left[M^{11}\right]\right\rangle, \quad\left|\chi^{(3)}(\theta)\right\rangle=\frac{1}{\sin \theta}\left|\left[M^{21}\right]\right\rangle
$$

and corresponding eigenvalues

$$
\lambda_{1}(\theta)=\cos ^{2}(\theta), \quad \lambda_{3}(\theta)=\sin ^{2}(\theta)
$$

For $0<\theta<\pi / 2$, we can then write

$$
\begin{gathered}
\omega_{\theta^{\prime}, 0}=\sum_{a=1}^{2} \Lambda_{a}^{\dagger}\left(\theta^{\prime}, \theta\right) \omega_{\theta, 0} \Lambda_{a}\left(\theta^{\prime}, \theta\right), \\
\Lambda_{a}\left(\theta^{\prime}, \theta\right)=\left(\frac{\lambda_{a}\left(\theta^{\prime}\right)}{\lambda_{a}(\theta)}\right)^{1 / 2}\left|\chi_{a}(\theta)\right\rangle\left\langle\chi_{a}\left(\theta^{\prime}\right)\right| .
\end{gathered}
$$

This makes sense for $\theta=0(\pi / 2)$ if the $a=2(a=1)$ term in $(\mathrm{V} .12)$ is understood as zero.

But positive maps cannot increase the rank of a state. Hence we cannot write evolution starting from $\theta=0$ or $\pi / 2$ in terms of positive maps.

\section{ANOMALIES AND RESTRICTIONS}

In this paper, mixed states emerge from restrictions of pure states $\omega$ on an algebra $\mathcal{A}$ to a subalgebra $\mathcal{A}_{0}$.

In a series of recent papers [19, 20], mixed states were introduced to eliminate anomalies. There it was proposed that anomalies can be eliminated by averaging say a pure state $\omega$ over the anomalous group.

We will now argue that the averaged state in the second case can also be regarded as the restriction of $\omega$ to a subalgebra.

Let us focus on parity anomaly caused for example by QCD $\theta$-angle. The discussion is valid for any $\mathbb{Z}_{2}$ symmetry group though.

Let $\mathcal{A}$ be the algebra of observables with unity $\mathbb{1}$. If $P$ is parity, then $\mathcal{A}$ has the parity-even subalgebra

$$
\mathcal{A}_{+}=\left\{a_{+} \in \mathcal{A}: \quad P a_{+} P=a_{+}\right\}
$$


and also its complement

$$
\mathcal{A}_{-}=\left\{a_{-} \in \mathcal{A}: P a_{-} P=-a_{-}\right\}
$$

which is not an algebra. The unity $\mathbb{1}$ is clearly in $\mathcal{A}_{+}$. But we assume that $\mathcal{A}_{+}$has its own unity (projector) $\mathbb{1}_{+}$:

$$
\mathbb{1}_{+} a_{+}=a_{+} \mathbb{1}_{+}=a_{+}, \quad \mathbb{1}_{+} a_{-}=a_{-} \mathbb{1}_{+}=0 .
$$

There is good physical meaning in assuming that $\mathcal{A}_{+}$contains both $\mathbb{1}_{+}$and $\mathbb{1}$. The projector $\mathbb{1}_{+}$is the unity on the parity even elements. We need its orthogonal projector as observable to tell us that the state of the system certainly has no component in $\mathcal{A}_{+}$. This orthogonal projector is $\mathbb{1}_{-}$. And $\mathbb{1}=\mathbb{1}_{+}+\mathbb{1}_{-}$.

Then

$$
\mathbb{1}_{-}=\mathbb{1}-\mathbb{1}_{+}
$$

is the projector onto $\mathcal{A}_{-}$:

$$
\mathbb{1}_{-} a_{+}=a_{+} \mathbb{1}_{-}=0, \mathbb{1}_{-} a_{-}=a_{-} \mathbb{1}_{-}=a_{-} .
$$

The parity-even subalgebra we consider is $\mathcal{A}_{0}=\mathcal{A}_{+} \oplus \mathbb{C} \mathbb{1}_{-}$

Let $\omega_{\theta}$, regarded as a density matrix, be such that

$$
P \omega_{\theta} P=\omega_{-\theta}
$$

For instance, a $\theta$-QCD state in QCD is of the above kind.

Since

$$
\mathbb{1}=\mathbb{1}_{+}+\mathbb{1}_{-},
$$

$\omega_{\theta}$ splits on restriction to $\mathcal{A}_{0}$ as

$$
\begin{aligned}
\omega_{\theta} & =\left|\left[\mathbb{1}_{+}\right]_{, \theta}\right\rangle\left\langle\left[\mathbb{1}_{+}\right]_{, \theta}|+|\left[\mathbb{1}_{-}\right]_{, \theta}\right\rangle\left\langle\left[\mathbb{1}_{-}\right]_{, \theta}\right| \\
& =\omega_{\theta,+}+\omega_{\theta,-} .
\end{aligned}
$$

Now consider the expectation value $\omega_{\theta}(\alpha)$ for

$$
\alpha=\alpha_{+}+\lambda \mathbb{1}_{-} \in \mathcal{A}_{0} .
$$

We have

$$
\omega_{\theta}(\alpha):=\operatorname{Tr} \omega_{\theta} \alpha=\omega_{\theta,+}\left(\alpha_{+}\right)+\omega_{\theta,-}\left(\lambda \mathbb{1}_{-}\right) .
$$


Since

$$
\begin{gathered}
P \alpha_{+} P=\alpha_{+}, \\
\omega_{\theta,+}\left(\alpha_{+}\right)=\omega_{-\theta,+}\left(\alpha_{+}\right)=\frac{1}{2}\left[\omega_{\theta,+}+\omega_{-\theta,+}\right]\left(\alpha_{+}\right) .
\end{gathered}
$$

Similarly since $P \mathbb{1}_{-} P=\mathbb{1}_{-}$,

$$
\omega_{\theta,-}=\frac{1}{2}\left[\omega_{\theta,-}+\omega_{-\theta,-}\right]
$$

Hence

$$
\left.\omega_{\theta}\right|_{\mathcal{A}_{0}}=\left.\frac{1}{2}\left(\omega_{\theta}+\omega_{-\theta}\right)\right|_{\mathcal{A}_{0}} .
$$

But

$$
\left(\omega_{\theta}+\omega_{-\theta}\right)\left(\alpha_{-}\right)=\omega_{\theta}\left(\alpha_{-}\right)+\omega_{\theta}\left(P \alpha_{-} P\right)=0,
$$

which is true of RHS of (VI.13) as well, extended to $\mathcal{A}_{-}$.

Hence restriction and averaging give same answer.

They will coincide whenever $\mathcal{A}_{0}$ has a projector, the analogue of $\mathbb{1}_{+}$here. This is clear from the computations above. Note that the normalisation of vectors in (VI.8) is given by

$$
\left\langle\left[\mathbb{1}_{ \pm}\right]_{, \theta} \mid\left[\mathbb{1}_{ \pm}\right]_{, \theta}\right\rangle=\omega_{\theta}\left(\mathbb{1}_{ \pm}\right) .
$$

\section{STATE RESTRICTIONS AND QUANTUM OBSERVATIONS}

Suppose we study the restriction of a state $\omega$ on an algebra $\mathcal{A}$ of observables to a subalgebra $\mathcal{A}_{0}$. For reasons explained in section VI, we assume that $\mathcal{A}_{0}$ contains the projector $\mathbb{1}_{+}$and its orthogonal projector

$$
\mathbb{1}_{-}=\mathbb{1}-\mathbb{1}_{+}
$$

$\mathbb{1}$ being the unity of $\mathcal{A}$. The projectors satisfy

$$
\mathbb{1}_{+} \mathbb{1}_{-}=0
$$

Using these projectors, we can decompose $\mathcal{A}_{0}$ into two parts:

$$
\mathcal{A}_{0}=\mathcal{A}_{0} \mathbb{1}_{+} \oplus \mathcal{A}_{0} \mathbb{1}_{-} .
$$

Let $\omega$ be pure on $\mathcal{A}$. Observe that $\mathcal{A}_{0} \mathbb{1}_{+}, \mathcal{A}_{0} \mathbb{1}_{-}$are both invariant by $\mathcal{A}_{0}$. Then, in the GNS construction, the restricted state splits into two parts:

$$
\left.\omega\right|_{\mathcal{A}_{0}}=\left|\left[\mathbb{1}_{+}\right]\right\rangle\left\langle\left[\mathbb{1}_{+}\right]|+|\left[\mathbb{1}_{-}\right]\right\rangle\left\langle\left[\mathbb{1}_{-}\right]\right|
$$


and is not pure.

Can we interpret (VII.4) as emergent from observations?

The answer seems to be yes. If one measures the probability of finding either 1 or 0 for the observable $\mathbb{1}_{+}$on an ensemble with state $\omega$, the resultant state $\left.\omega\right|_{\mathcal{A}_{0}}$ is after measurement exactly (VII.4):

$$
\left.\omega \rightarrow \omega\right|_{\mathcal{A}_{0}}=\mathbb{1}_{+}|[\mathbb{1}]\rangle\left\langle[\mathbb{1}]\left|\mathbb{1}_{+}+\mathbb{1}_{-}\right|[\mathbb{1}]\right\rangle\langle[\mathbb{1}]| \mathbb{1}_{-}
$$

In this case, we are given the projector $\mathbb{1}_{+}$and we can reconstruct $\mathcal{A}_{0} \subseteq \mathcal{A}$ as its commutant:

$$
\mathcal{A}_{0}=\text { Commutant of } \mathbb{1}_{+} \text {in } \mathcal{A} \text {. }
$$

Working from this $\mathcal{A}_{0}$, we then show that the state restricted to (VII.6) coincides with VII.5).

\section{CONCLUSIONS}

We have seen in this work that there is a natural formulation of quantum physics dispensing with the use of Hilbert space as initial data which is well-adapted to the study of entanglement and entropy. In this approach, Hilbert space is an emergent concept. Instead, the initial data are the algebra of observables and their expectation values. From the expectation values one abstracts the notion of a state on the algebra.

In this formulation, the Hilbert space is obtained from the GNS construction that resembles the construction of the regular representation of finite (or compact) groups. Furthermore, one may compute the von Neumann entropy associated with a density matrix that is obtained from a state on the algebra. It should be emphasized that for each state one may associate many distinct density matrices and therefore distinct von Neumann entropies. The discussion of this point is carried out in [22].

A state $\omega$ on an algebra $\mathcal{A}$ can be restricted to a subalgebra $\mathcal{A}_{0}$. The new state $\left.\omega\right|_{\mathcal{A}_{0}}$ may not be pure even if $\omega$ is. Its entropy is a measure of entanglement of $\mathcal{A}_{0}$ with $\mathcal{A}$.

This new approach to entanglement lets us treat identical particles obeying Bose, Fermi or even braid statistics with ease. Particle identity has posed severe problems in conventional approaches.

We have also shown how time evolution by positive maps for $\left.\omega\right|_{\mathcal{A}_{0}}$ emerges when $\omega$ evolves unitarily. 
Further points we have treated concerning quantum anomalies and their elimination by restricting states to subalgebras. In this manner, we can understand the use of mixed states to eliminate anomalies suggested by our previous work [19]. We also discussed how the restriction $\left.\omega\right|_{\mathcal{A}_{0}}$ emerges from a standard interpretation of quantum physics from the observation of projectors.

\section{ACKNOWLEDGMENTS}

The authors would like to thank Alonso Botero for discussions that led to this work. We also thank M. Asorey, B. Carneiro da Cunha, S. Ghosh, K. Gupta, A. Ibort, G. Marmo, V. P. Nair and A. Pinzul for fruitful discussions during different stages of this work. APB is supported by the Institute of Mathematical Sciences, Chennai. ARQ is supported by CNPq under process number 307760/2009-0. AFRL is supported by Universidad de los Andes.

[1] R. Haag, Local quantum physics (Text and Monographs in Physics, 2nd ed., Springer, 1996).

[2] A. P. Balachandran, T. R. Govindarajan, A. R. de Queiroz, and A. F. Reyes-Lega, Phys. Rev. Lett. (in press) (2013), 1205.2882.

[3] Y. S. Li, B. Zeng, X. S. Liu, and G. L. Long, Phys. Rev. A 64, 054302 (2001).

[4] R. Paskauskas and L. You, Phys. Rev. A 64, 042310 (2001).

[5] J. Schliemann, J. I. Cirac, M. Kuś, M. Lewenstein, and D. Loss, Phys. Rev. A 64, 022303 (2001).

[6] P. Zanardi, Phys. Rev. A 65, 042101 (2002).

[7] A. Fang and Y. Chang, Physics Letters A 311, 443 (2003).

[8] G. Ghirardi and L. Marinatto, Phys. Rev. A 70, 012109 (2004).

[9] P. Lévay, S. Nagy, and J. Pipek, Phys. Rev. A 72, 022302 (2005).

[10] M.-C. Bauls, J. I. Cirac, and M. M. Wolf, Journal of Physics: Conference Series 171, 012032 $(2009)$.

[11] A. Plastino, D. Manzano, and J. Dehesa, European Physics Letters 86, 20005 (2009).

[12] C. Zander, A. Plastino, M. Casas, and A. Plastino, The European Physical Journal D Atomic, Molecular, Optical and Plasma Physics 66, 1 (2012), 10.1140/epjd/e2011-10654-x. 
[13] H. Barnum, E. Knill, G. Ortiz, S. Rolando, and L. Viola, Physical Review Letters 92, 107902 (2004).

[14] F. Benatti, R. Floreanini, and U. Marzolino, Annals of Physics 327, 1304 (2012).

[15] N. L. Harshman and K. S. Ranade, Phys. Rev. A 84, 012303 (2011).

[16] Ł. Derkacz, M. Gwóźdź, and L. Jakóbczyk, Journal of Physics A: Mathematical and Theoretical 45, 025302 (2012).

[17] A. P. Balachandran, S. G. Jo, and G. Marmo, Group Theory and Hopf Algebras: Lectures for Physicists (World Scientific, 2010).

[18] W. F. Stinespring, Proc. Amer. Math. Soc. 6, 211 (1955).

[19] A. P. Balachandran and A. R. de Queiroz, Phys. Rev. D 85, 025017 (2012).

[20] A. P. Balachandran, T. R. Govindarajan, and A. Queiroz, The European Physical Journal Plus 127, 1 (2012).

[21] Private communication.R. D. Sorkin, (2012), arXiv:1205.2953 [hep-th]

[22] A. P. Balachandran, A. R. de Queiroz, and S. Vaidya, (2012), arXiv:1212.1239 [hep-th],

[23] G. Landi, An Introduction to Noncommutative Spaces and their Geometry (vol. 51, Lecture Notes in Physics Monographs, Springer-Verlag, 2008).

[24] C. Aneziris, A. P. Balachandran, M. Bourdeau, S. Jo, T. R. Ramadas, and R. D. Sorkin, Int.J.Mod.Phys. A4, 5459 (1989).

[25] C. Aneziris, A. P. Balachandran, L. Kauffman, and A. M. Srivastava, Int.J.Mod.Phys. A6, 2519 (1991).

[26] A. P. Balachandran and A. R. de Queiroz, JHEP 1111, 126 (2011), arXiv:1109.5290 [hep-th],

[27] M. C. Tichy, F. Mintert, and A. Buchleitner, Journal of Physics B: Atomic, Molecular and Optical Physics 44, 192001 (2011).

[28] L. C. Biedenharn and M. A. Lohe, Quantum Group Symmetry and q-Tensor Algebras (World Scientific, 1995). 